For numbered affiliations see end of article.

Correspondence for this iteration to: François Lamontagne

francois.lamontagne@usherbrooke.ca; Miriam Stegemann

miriam.stegemann@charite.de.

Additional material is published online only. To view please visit the journal online

Cite this as: BMJ 2020;370:m3379 http://dx.doi.org/10.1136/bmj.m3379

RAPID RECOMMENDATIONS

\title{
A living WHO guideline on drugs for covid-19
}

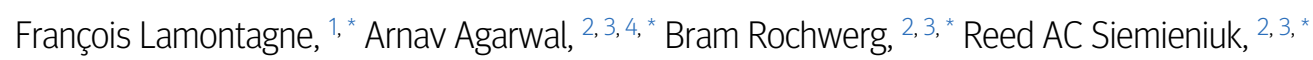
Thomas Agoritsas, $2,4,5,{ }^{*}$ Lisa Askie, ${ }^{6,{ }^{*}}$ Lyubov Lytvyn, $2,{ }^{*}$ Yee-Sin Leo, ${ }^{7}$ Helen Macdonald, ${ }^{8,}{ }^{*}$ Linan Zeng, ${ }^{2}$ * Wagdy Amin, ${ }^{9}$ André Ricardo Araujo da Silva, ${ }^{10}$ Diptesh Aryal, ${ }^{11}$ Fabian A Jaimes Barragan, ${ }^{12}$ Frederique J Bausch, ${ }^{13}$ Erlina Burhan, ${ }^{14}$ Carolyn S Calfee, ${ }^{15}$ Maurizio Cecconi, ${ }^{16}$ Binila Chacko, ${ }^{17}$ Duncan Chanda, ${ }^{18}$ Vu Quoc Dat, ${ }^{19}$ An De Sutter, ${ }^{20}$ Bin Du, ${ }^{21}$ Stephen Freedman, ${ }^{22}$ Heike Geduld, ${ }^{23}$ Patrick Gee, ${ }^{24}$ Matthias Gotte, ${ }^{25}$ Nerina Harley, ${ }^{26}$ Madiha Hashmi, ${ }^{27}$ Beverley Hunt, ${ }^{28}$ Fyezah Jehan, ${ }^{29}$ Sushil K Kabra, ${ }^{30}$ Seema Kanda, ${ }^{31}$ Yae-Jean Kim, ${ }^{32}$ Niranjan Kissoon, ${ }^{33}$ Sanjeev Krishna, ${ }^{34}$ Krutika Kuppalli, ${ }^{6}$ Arthur Kwizera, ${ }^{35}$ Marta Lado Castro-Rial, $6,{ }^{*}$ Thiago Lisboa, ${ }^{36}$ Rakesh Lodha, ${ }^{37}$ Imelda Mahaka, ${ }^{38}$ Hela Manai, ${ }^{39}$ Marc Mendelson, ${ }^{40}$ Giovanni Battista Migliori, ${ }^{41}$ Greta Mino, ${ }^{42}$ Emmanuel Nsutebu, ${ }^{43}$ Jacobus Preller, ${ }^{6,}{ }^{*}$ Natalia Pshenichnaya, ${ }^{44}$ Nida Qadir, ${ }^{45}$ Pryanka Relan, ${ }^{6,}$ ' Saniya Sabzwari, 46 Rohit Sarin, ${ }^{47}$ Manu Shankar-Hari, ${ }^{48}$ Michael Sharland, ${ }^{49}$ Yinzhong Shen, ${ }^{50}$ Shalini S Ranganathan, ${ }^{5}$ Joao P Souza, ${ }^{52}$ Miriam Stegemann, ${ }^{53}$ Ronald Swanstrom, ${ }^{54}$ Sebastian Ugarte, ${ }^{55}$ Tim Uyeki, ${ }^{56}$ Sridhar Venkatapuram, ${ }^{57}$ Dubula Vuyiseka, ${ }^{58}$ Ananda Wijewickrama, ${ }^{59}$ Lien Tran, ${ }^{60},{ }^{*}$ Dena Zeraatkar, ${ }^{2}$

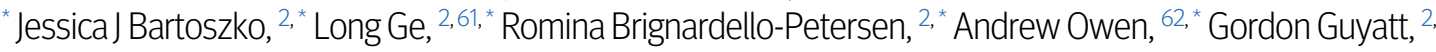
3, a, * Janet Diaz, 6, a, * Leticia Kawano-Dourado, ${ }^{63}$ Michael Jacobs, ${ }^{64, \text { a }}$ Per Olav Vandvik4, 65, a, *

\section{ABSTRACT UPDATES}

This is the thirteenth version (twelfth update) of the living guideline, replacing earlier versions (available as data supplements). New recommendations will be published as updates to this guideline. CLINICAL QUESTION

What is the role of drugs in the treatment of patients with covid-19?

\section{CONTEXT}

The evidence base for therapeutics for covid-19 is evolving with numerous randomised controlled trials (RCTs) recently completed and under way. Emerging SARS-CoV-2 variants (such as omicron) and subvariants are changing the role of therapeutics. This update does not include any changes to the strength or direction of recommendations, but rather concerns i) the use of nirmatrelvir/ritonavir, now considered to be an option also for pregnant and breastfeeding women with non-severe covid-19, and ii) evidence of reduction of in vitro neutralisation activity supporting the strong recommendations against the use of the neutralising monoclonal antibodies sotrovimab and casirivimab-imdevimab. RECOMMENDATIONS, UNCHANGED FROM PREVIOUS

- Recommended for patients with severe or critical covid-19-strong recommendations for systemic corticosteroids, IL-6 receptor blockers (tocilizumab or sarilumab) in combination with corticosteroids, and baricitinib as an alternative to IL-6 receptor blockers, in combination with corticosteroids. Concerning the concomitant use of IL- 6 receptor blockers (tocilizumab and sarilumab), and the JAK inhibitor baricitinib, these drugs may be combined, in addition to corticosteroids.

- Recommended for patients with severe covid-19-a conditional recommendation for remdesivir.
- Not recommended for patients with critical covid-19-a conditional recommendation against remdesivir.

- Recommended for patients with non-severe covid-19 at highest risk of hospitalisation-a strong recommendation for nirmatrelvir/ritonavir; conditional recommendations for molnupiravir and remdesivir.

- Not recommended for patients with non-severe covid-19-a conditional recommendation against systemic corticosteroids and colchicine; a strong recommendation against convalescent plasma; a recommendation against fluvoxamine, except in the context of a clinical trial.

- Not recommended for patients with non-severe covid-19 at low risk of hospitalisation-a conditional recommendation against nirmatrelvir/ritonavir.

- Not recommended for patients with severe or critical covid-19-a recommendation against convalescent plasma except in the context of a clinical trial; a conditional recommendation against the JAK inhibitors ruxolitinib and tofacitinib.

- Not recommended, regardless of covid-19 disease severity-strong recommendations against

hydroxychloroquine, lopinavir/ritonavir, sotrovimab, and casirivimab-imdevimab; and a recommendation against ivermectin except in the context of a clinical trial.

\section{ABOUT THIS GUIDELINE}

This living guideline from the World Health Organization (WHO) incorporates new evidence to dynamically update recommendations for covid-19 therapeutics. The Guideline Development Group (GDG) typically evaluates a therapy when the WHO judges sufficient evidence is available to make a recommendation. While the GDG takes an individual patient perspective in making recommendations, it also considers resource implications, acceptability, feasibility, equity, and human rights. This guideline 
was developed according to standards and methods for trustworthy guidelines, making use of an innovative process to achieve efficiency in dynamic updating of recommendations. The methods are aligned with the WHO Handbook for Guideline Development and according to a pre-approved protocol (planning proposal) by the Guideline Review Committee (GRC). A box at the end of the article outlines key methodological aspects of the guideline process. MAGIC Evidence Ecosystem Foundation provides methodological support, including the coordination of living systematic reviews with network meta-analyses to inform the recommendations. The full version of the guideline is available online in MAGICapp and in PDF, with a summary version here in The BMJ. These formats should facilitate adaptation, which is strongly encouraged by WHO to contextualise recommendations in a healthcare system to maximise impact.

\section{FUTURE RECOMMENDATIONS}

Recommendations on anticoagulation and updated recommendations on molnupiravir are planned for the next updates to this guideline.

Vaccines are linked to limiting hospitalisations, but limitations in global access and residual uncertainties remain regarding the duration of protection following vaccination or natural infection, and their efficacy against emerging SARS-CoV-2 variants and subvariants. Therefore, the potential for drugs to treat people infected with covid-19 remains of interest. This living guideline responds to emerging evidence from randomised controlled trials (RCTs) on existing and new drug treatments for covid-19.

More than 5000 trials investigating covid-19 interventions have been registered or are ongoing (see section on emerging evidence ${ }^{1}$ ). Although most of these studies are small and of variable methodological quality, some large, international platform trials have provided robust evidence. Such trials can also adapt their design, recruitment strategies, and selection of interventions based on new insights. Examples include ACCT, RECOVERY, WHO

SOLIDARITY, REMAP-CAP, and ACTIV, which recruit large numbers of patients in many countries. ${ }^{2-5}$ An overview of ongoing trials is available from the Infectious Diseases Data Observatory, through their living systematic review of covid-19 clinical trial registrations ${ }^{1}$ and the World Health Organization (WHO) website

(https://www.covid-nma.com/dataviz/).

However, existing and emerging evidence demonstrates remaining uncertainties concerning treatment effects for all outcomes of importance to patients. There is also a need for better evidence on values and preferences of patients with covid-19. Moreover, the rapidly evolving evidence landscape requires trustworthy

interpretation and expeditious clinical practice guidelines to inform clinicians and health care decision-makers.

Several living network meta-analyses associated with this guideline incorporate emerging trial data and allow for analysis of comparative effectiveness of multiple covid-19 treatments. ${ }^{67}$ Box 1 includes these network meta-analyses and other related publications. To inform the living guidance, we also use additional relevant evidence on safety, prognosis, and patient values and preferences related to covid-19 treatments.

\section{Box 1: Linked resources in this BMJ Rapid Recommendations cluster} Versions of this guidance

- This article and infographic: Lamontagne F, Agarwal A, Rochwerg B, et al. A living WHO guideline on drugs for covid-19 [Update 12, published January 2023]. BM/ 2020;370:m3379,

doi:10.1136/bmj.m3379
- WHO PDF: World Health Organization. Therapeutics and COVID-19. Living guideline. January 2023. https://www.who.int/teams/healthcare-readiness-clinical-unit/covid-19/therapeutics

- MAGICapp (https://app.magicapp.org/\#/guideline/nBkO1E)

- Expanded version of the guideline, including methods, processes, and results with multi-layered recommendations, evidence summaries, and decision aids for use on all devices

MATCH-IT interactive decision support incorporating multiple treatment comparisons for recommended drugs in non-severe covid-19 at highest risk of hospitalisation: https://magicevidence.org/match-it/220404dist-covid-meds/

\section{Linked research}

- Siemieniuk RAC, Bartoszko JJ, Ge L, et al. Drug treatments for covid-19: living systematic review and network meta-analysis [Update 4, published June 2022]. BM/ 2020;370:m2980, doi:10.1136/bmj.m2980

Updated evidence available online: https://www.covid19lnma.com/

- Siemieniuk RAC, Bartoszko JJ, Díaz Martinez JP, et al. Antibody and cellular therapies for treatment of covid-19: a living systematic review and network meta-analysis. BMJ 2021;374:n2231, doi:10.1136/bmj.n2231

- Zeraatkar D, Cusano E, Diaz Martinez JP, et al. Tocilizumab and sarilumab alone or in combination with corticosteroids for COVID-19: a systematic review and network meta-analysis. medRxiv 2021; doi:10.1101/2021.07.05.21259867v1

- Izcovich A, Siemieniuk RAC, Bartoszko JJ, et al. Adverse effects of remdesivir, hydroxychloroquine, and lopinavir/ritonavir when used for COVID-19: systematic review and meta-analysis of randomized trials. medRxiv 2020; doi:10.1101/2020.11.16.20232876v1

- Lamontagne F, Agoritsas T, Siemieniuk R, et al. A living WHO guideline on drugs to prevent covid-19. BMJ 2021;372:n526. doi:10.1136/bmj.n526

- World Health Organization. Clinical management of COVID-19: interim guidance. 2020. https://www.who.int/publications/i/item/clinicalmanagement-of-covid-19

- Wynants L, Van Calster B, Collins GS, et al. Prediction models for diagnosis and prognosis of covid-19: systematic review and critical appraisal. BMJ 2020;369:m1328

\section{What triggered this version of the guideline and what is coming next?}

This thirteenth version of the WHO living guideline was triggered by

- Availability of data providing evidence that serious adverse reactions may be very rare with nirmatrelvir/ritonavir in pregnant or breastfeeding women;

- Availability of additional evidence from high quality in vitro neutralisation experiments suggesting that monoclonal antibodies sotrovimab and casirivimab-imdevimab will have little or no benefit in circulating omicron variants.

The WHO has a standing steering committee to evaluate possibilities for new drug recommendations and updates to existing drug recommendations. The WHO considers multiple factors, including the extent of available evidence, and whether and when additional evidence might be anticipated, to make decisions. Other therapeutics in progress for this WHO living guideline include therapeutic anticoagulation and updated recommendations for molnupiravir. 


\section{How to use this guideline and associated resources}

This is a living guideline. The recommendations and evidence included here will be updated, and new recommendations will be added for other treatments for covid-19. The infographic provides a summary of the recommendations. Readers can find more detailed information in the full version of the WHO guideline (see box 1 for links to MAGICapp and the PDF version).

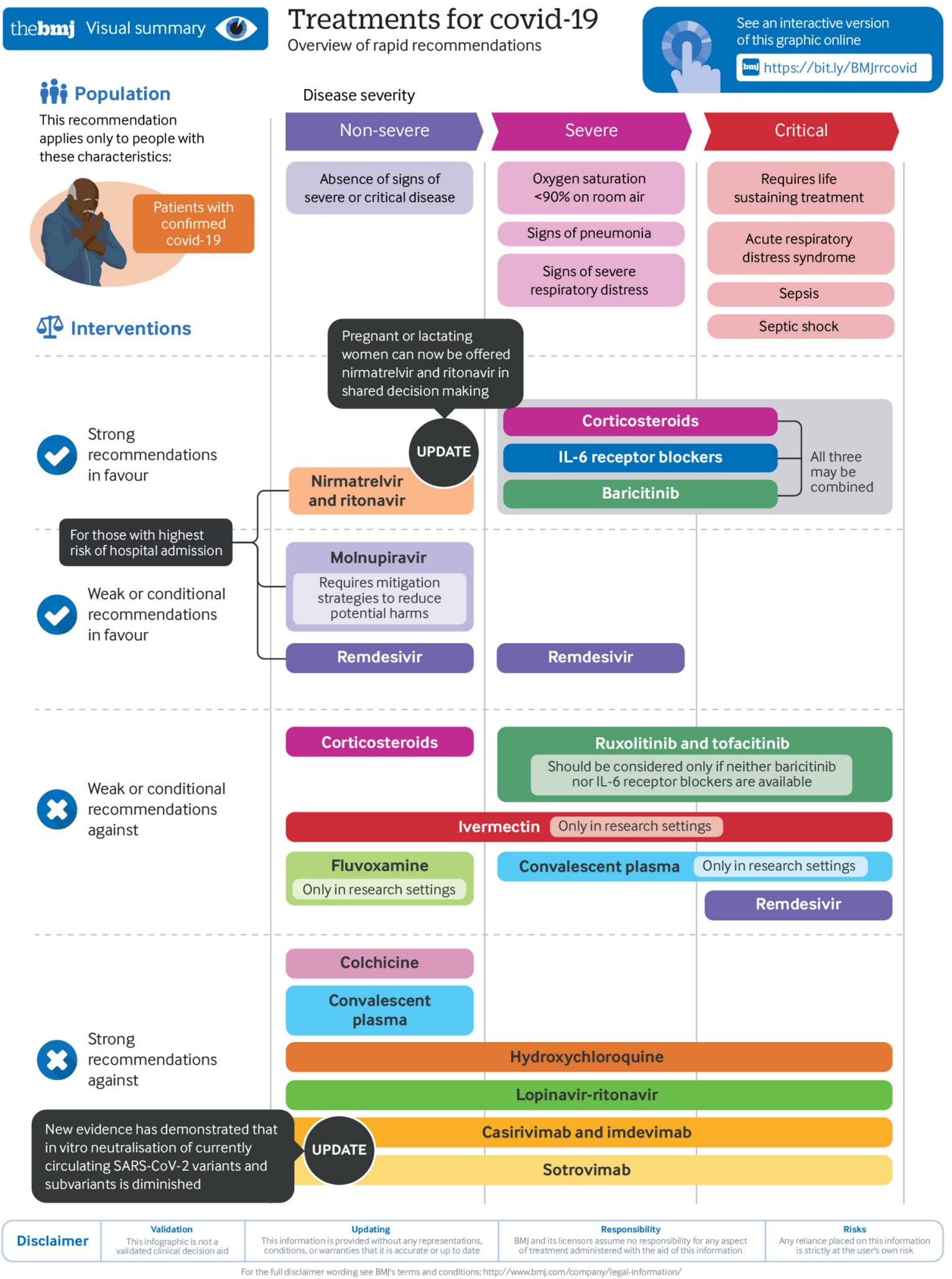




\section{Who do the recommendations apply to?}

This guideline applies to all patients with covid-19.

Recommendations may differ based on the severity of covid-19, according to WHO severity definitions (box 2). ${ }^{8}$ These definitions avoid reliance on access to healthcare to define patient subgroups.

\section{Box 2: WHO definitions of illness severity for covid-19}

- Critical covid-19-Defined by the criteria for acute respiratory distress syndrome (ARDS), sepsis, septic shock, or other conditions that would normally require the provision of life-sustaining therapies such as mechanical ventilation (invasive or non-invasive) or vasopressor therapy.

- Severe covid-19-Defined by any of:

Oxygen saturation $<90 \%$ on room air*

Signs of pneumonia

Signs of severe respiratory distress (in adults, accessory muscle use, inability to complete full sentences, respiratory rate > 30 breaths per minute; and, in children, very severe chest wall indrawing, grunting, central cyanosis, or presence of any other general danger signs including inability to breastfeed or drink, lethargy, convulsions, or reduced level of consciousness).

- Non-severe covid-19-Defined as the absence of any criteria for severe or critical covid-19.

*The Guideline Development Group (GDG) noted that the oxygen saturation threshold of $90 \%$ to define severe covid-19 was arbitrary and should be interpreted cautiously when defining illness severity. For example, clinicians must use their judgment to determine whether a low oxygen saturation is a sign of severity or is normal for a given patient with chronic lung disease. Similarly, clinicians may interpret a saturation of $90-94 \%$ on room air as abnormal in a patient with normal lungs, or as an early sign of severe disease in a patient with a downward clinical trajectory. Generally, in cases where there is any doubt, the GDG suggested erring on the side of considering disease as severe.

\section{How to use the recommendations}

\section{Selecting therapeutic agents}

Over two years into the pandemic, there are several effective treatments to choose between. When moving from evidence to recommendations for these drugs, the Guideline Development Group (GDG) considered a combination of the evidence regarding relative benefits and harms, values and preferences, practical issues, resource considerations, and feasibility and equity considerations (box 3). The GDG notes that these issues have to be considered when choosing between therapeutic agents, and when re-using or adapting the recommendations in national or local contexts.

\section{Box 3: Resources, access, and equity issues when choosing therapeutics}

Several drugs may be unavailable or impractical for use in some contexts. Additional obstacles to access in low and middle income countries (LMICs) may include cost and availability, and limited access to services such as diagnostic testing and treatments within the first five days of symptoms, which may further limit access to interventions. Health inequities may be exacerbated if patients at higher risk receive the intervention. See the full version of the guideline (box 1) for more information.

WHO aims to provide a stimulus to engage all possible mechanisms to improve global access to diagnostic tests and effective interventions and how countries can address such challenges; such as the integration of a covid-19 clinical care pathway and establishing services to offer oral and intravenous treatments.

At a time of drug shortage, it may be necessary to prioritise use through clinical triage such as selecting patients with the highest baseline risk for mortality, in whom the absolute benefit of treatment is greatest. Other suggestions for prioritisation, which lack direct evidence, include focusing on patients with an actively deteriorating clinical course and avoiding treatment in patients with established multi-organ failure (in whom the benefit is likely to be small).

Some therapies can be used in combination (such as those for severe or critical covid-19) while others are to be used as alternatives. Recommended combinations of treatments are based on direct comparisons from trials demonstrating additional benefit, such as adding the JAK inhibitor baricitinib to IL-6 receptor blockers and to systemic corticosteroids in patients with severe or critical covid-19.

An interactive decision support tool incorporates multiple treatment comparisons to inform the use of one drug over another with related mechanisms of action for patients with non-severe covid-19 at highest risk of hospitalisation: https://magicevidence.org/matchit/220404dist-covid-meds/.

\section{Identifying patients with non-severe illness at highest risk of hospitalisation}

Several recommendations for therapeutics in patients with non-severe covid-19 apply only to those at highest risk of hospitalisation, defined as beyond $10 \%$ risk of being hospitalised for covid-19. These patients should achieve what the GDG agreed would represent what most patients would value as an important benefit: a $6 \%$ absolute reduction in hospital admission (see box on how this guideline was created). Reliably identifying those at highest risk is challenging because of the changing global context, with evolution of the virus and patterns of vaccination, thus raising the importance of validation of models to local contexts. A living systematic review of 232 risk prediction models for covid-19, recently updated, identified two promising risk prediction tools before circulation of the omicron variant: these tools concur that typical characteristics of people at highest risk include older age, immunosuppression, and/or presence of chronic diseases, with lack of vaccination as an additional risk factor. ${ }^{9}$

\section{Uncertainties}

Uncertainties exist regarding covid-19 therapeutics and emerging evidence which may both inform clinical decision-making as well as future research. The recommendations therefore need to be used in light of these uncertainties. Specific uncertainties are listed with the relevant drug, but many uncertainties are common across therapeutics:

- For drugs recommended in non-severe illness: the lack of accurate clinical prediction guides to establish the individual patient risk of hospitalisation in order to best identify patients that would most benefit from interventions; data regarding emergence of resistance and efficacy against new variants; safety and efficacy in children and in immunocompromised, vaccinated, or pregnant patients and other specific subgroups of patients; optimal duration of therapies; head-to-head comparisons of recommended treatments; and relative effectiveness of combination therapy and longer term outcomes.

- For drugs recommended in severe or critical illness: safety and efficacy in children and in immunocompromised, vaccinated, or pregnant patients and other specific subgroups of patients; long term mortality and functional outcomes in covid-19 survivors; and immunity and the risk of a subsequent infection, which may affect the risk of death after 28 days. 


\section{The recommendations}

Nirmatrelvir/ritonavir (Update 12, published 13 January 2023)

\section{Overview}

Nirmatrelvir is a SARS-CoV protease inhibitor which prevents viral replication. Nirmatrelvir/ritonavir is administered orally in combination with ritonavir, a HIV protease inhibitor, which improves its pharmacokinetics. Through its impact on metabolism and clearance, ritonavir is a perpetrator of many drug-drug interactions during active treatment and possibly for several days after treatment completion. Although these may be more easily managed with short durations of treatment, twice daily administration involves doubling ritonavir dose relative to most modern antiretroviral regimens.

In vitro animal studies and human models have demonstrated the antiviral effect of nirmatrelvir. ${ }^{10-12}$ Nirmatrelvir retains activity against all SARS-CoV-2 lineages studied in vitro to date, ${ }^{13}$ but clinical data are currently unavailable. ${ }^{14}$ There remains uncertainty regarding risk of emergence of resistance; in vitro studies have suggested acquired mutations in the protease sequence may significantly reduce nirmatrelvir activity, though variably so depending on type and number of mutations. ${ }^{10}$

Update-An initial strong recommendation for patients with non-severe covid-19 at highest risk of hospitalisation, and a conditional recommendation against use of nirmatrelvir/ritonavir for patients at low risk of hospitalisation, were published on 22 April 2022 as the tenth version of the living guideline. This was based on data from two RCTs available at the time.

In the thirteenth iteration of the guideline, an updated recommendation was made concerning the use of nirmatrelvir/ritonavir in breastfeeding and pregnant women with non-severe illness, based on data mainly available through the WHO Vigibase (https://who-umc.org/vigibase). The recommendation now includes a statement that breastfeeding and pregnant women with non-severe covid-19 may consider use of nirmatrelvir/ritonavir.

Evidence - There were no reported serious adverse events linked to nirmatrelvir/ritonavir in pregnant or breastfeeding women-either in mother or child; however, there was residual uncertainty pertaining to the denominator to which this estimate of no undesirable effects applied. Therefore, given the likely benefits and residual uncertainty regarding undesirable effects, the

recommendation was updated to reflect the GDG's belief that shared, fully informed decision-making between mother and healthcare provider should determine the use or non-use of nirmatrelvir/ritonavir in pregnant or breastfeeding women with non-severe covid-19.

See MAGICapp for detailed description of the mechanism of action, evidence, and subgroup analyses underpinning the recommendations, and regarding data from the WHO Vigibase pertaining to breastfeeding and pregnant women (https://app.magicapp.org/\#/guideline/nBkO1E/rec/LwrMyv and https://app.magicapp.org/\#/guideline/nBkO1E/rec/Lrvo3R).

Recommendation 1: For patients with non-severe covid-19 at highest risk of hospitalisation, we recommend treatment with nirmatrelvir/ritonavir (strong recommendation).

\section{Understanding the recommendation}

Moderate certainty evidence of a substantial relative risk reduction in hospitalisation, and high certainty evidence of no adverse effects requiring drug discontinuation, motivated the strong recommendation in individuals at higher risk of hospitalisation. Such individuals are likely to achieve an important reduction in the absolute risk of hospitalisation in comparison with those not receiving nirmatrelvir/ritonavir.

Only a min ority of patients who are at highest risk are likely to achieve sufficient benefit in reduced admission to hospital. Nirmatrelvir/ritonavir likely represents a superior choice for these patients because it may have greater efficacy in preventing hospitalisation than available alternatives, has fewer concerns with respects to harms than molnupiravir, and is easier to administer than intravenous remdesivir and antibodies.

With regards to alternative or combination therapy, the GDG has previously made a conditional recommendation for molnupiravir and remdesivir in the highest risk non-severe population. Indirect comparisons in higher and highest risk patients found

nirmatrelvir/ritonavir may reduce hospitalisation when compared with molnupiravir (low certainty); however, they found little or no difference when compared with remdesivir (low certainty). Without direct data comparisons and low certainty confidence in indirect comparisons, the GDG chose not to make comparative recommendations between drugs, but rather remark that nirmatrelvir/ritonavir may be superior based on its efficacy compared with standard care (moderate certainty) and that, ultimately, choice of therapeutic may be made based on practical issues such as ease of administration and risk profiles. There is no evidence for combining antiviral therapies; the GDG therefore advised against this.

Balance of benefits and harms-Beyond the important benefits in reducing hospital admission in patients at highest risk of hospital admission, treatment may have little or no impact on mortality; data regarding effects on time to symptom resolution or mechanical ventilation are limited. Treatment does not increase the likelihood of serious adverse effects leading to drug discontinuation, though diarrhoea and altered taste were noted to occur more often with treatment relative to placebo. The GDG also acknowledged the paucity of information related to emergence of resistance.

Values and preferences-The GDG inferred that almost all well informed patients at highest risk of hospitalisation would choose to receive nirmatrelvir/ritonavir.

Applicability-Because pregnancy represents a risk factor for severe or critical illness in those with non-severe covid-19, pregnant women might consider using medication that reduces the risk of disease progression. Nirmatrelvir/ritonavir, the drug combination the WHO recommends most highly in the context of non-severe illness for patients at highest risk of hospitalisation, represents a possible option.

Nevertheless, as with any medication not formally tested in pregnancy, concerns regarding undesirable effects in both mother and fetus immediately arise. Data from the WHO Vigibase, a comprehensive collection of worldwide unpublished reports of possible adverse reactions to drugs can inform the issue of undesirable effects.

Up to now, there have been no reports linking nirmatrelvir/ritonavir to serious adverse reactions in pregnant or breastfeeding women, either in mother or child. This is reassuring, but only to an extent: we are uncertain of the denominator to which this estimate of no undesirable effects applies. If a large number of women have been exposed, the absence of reported undesirable effects provides 
considerable reassurance; if only a small number, not so. We are uncertain which is the case.

In providing guidance on nirmatrelvir/ritonavir use in pregnancy, the GDG considered the likely benefits (there is no reason to think the drug will be less effective in pregnant women than in other people) and the uncertainty regarding undesirable effects. The GDG believes that shared, fully informed decision making between mother and healthcare provider should determine the use of nirmatrelvir/ritonavir in pregnant or breastfeeding women with non-severe covid-19.

Practical issues-As per the large trials informing the recommendation, nirmatrelvir/ritonavir is administered as 300 $\mathrm{mg} / 100 \mathrm{mg}$ orally every 12 hours for five days. Administration should be as early as possible in the course of the disease. In the included studies, nirmatrelvir/ritonavir was administered within five days of disease onset. Dose reduction to $150 \mathrm{mg} / 100 \mathrm{mg}$ orally every 12 hours for five days may be warranted in cases of renal insufficiency with estimated glomerular function rates of 30-59 mL/min. Through its impact on metabolism and clearance, ritonavir is a perpetrator of many drug-drug interactions, warranting serious consideration by clinicians. The Liverpool covid-19 drug interaction checker may constitute a valuable tool for management of drug interactions with nirmatrelvir/ritonavir. ${ }^{15}$ Additional considerations regarding practical issues are summarised in MAGICapp.

Resource implications, acceptability, feasibility, equity, and human rights-Nirmatrelvir/ritonavir is unlikely to be available for all individuals who, given the option, would choose to receive the treatment. Since this recommendation is for treatment with nirmatrelvir/ritonavir within five days of symptom onset, access to and appropriate use of diagnostic tests are essential for implementation.

Recommendation 2: For patients with non-severe covid-19 at
low risk of hospitalisation, we suggest not to use
nirmatrelvir/ritonavir (conditional or weak recommendation). Understanding the recommendation

Most patients with non-severe covid-19 at low risk of hospitalisation will experience trivial benefits with use of nirmatrelvir/ritonavir. The GDG inferred that most such patients would be uninterested in taking the drug for these trivial benefits. However, there are likely to be an appreciable number of individuals who place a high value on very small reductions in the risk of hospitalisation and would thus choose to use nirmatrelvir/ritonavir; therefore, a conditional (rather than a strong) recommendation was made. The benefits and harms and other factors the GDG considered are comprehensively described in the full version of the guideline (see box 1).

Specific uncertainties, emerging evidence, and future research (across disease severities)

No drug-specific uncertainties: see broadly applicable uncertainties section in "How to use this guideline" (above).

Remdesivir (Update 11, published 16 September 2022)

\section{Overview}

Remdesivir was developed for treatment of hepatitis $C$ virus infection, and was also studied in Ebola and Marburg virus infections before being repurposed for SARS-CoV-2. Remdesivir is a nucleoside analogue which interacts with the SARS-CoV-2 polymerase to elicit delayed chain termination during RNA genome synthesis; its mechanism of action is further described in the full version of the guideline (box 1). Resistance to remdesivir has been reported in vitro and in a case report, but the clinical significance of these observations remain unclear.

An initial conditional (weak) recommendation was made on 20 November 2020, suggesting not to use remdesivir for patients with covid-19 regardless of illness severity. This was based on data from the four RCTs available at the time, with 7333 participants hospitalised for covid-19. In the tenth iteration of the guideline, a new recommendation was made for the use of remdesivir for patients with non-severe illness. In the twelfth iteration of the guideline, new recommendations for patients with severe or critical covid-19 were provided, given new trial data providing sufficiently trustworthy evidence for a subgroup effect demonstrating modest benefit in patients with severe, but not critical, covid-19.

Evidence-For patients with severe or critical covid-19, data were derived from five trials that enrolled 7643 patients. See MAGICapp for detailed description of the mechanism of action, evidence, and subgroup analyses underpinning the recommendation.

Recommendation 1: For patients with severe covid-19, we suggest treatment with remdesivir (weak or conditional recommendation).

\section{Understanding the recommendation}

When moving from evidence to the conditional recommendation to use remdesivir in patients with severe covid-19, the GDG emphasised the benefits on survival and reduction in need for invasive mechanical ventilation, and the likelihood of little or no serious adverse events attributable to the drug. The GDG acknowledged that some serious adverse events may not have been accurately captured during the relatively short follow-up period in the included trials. Of note, although the GDG has recommended for other antiviral drugs in patients with non-severe illness, remdesivir is the only one with a recommendation for use in patients with severe covid-19.

The GDG did not anticipate important variability in patient values and preferences, although the low certainty of evidence and ongoing uncertainty in effect contributed to the conditional recommendation. There was insufficient trial level data to examine subgroups based on age or to consider patients requiring non-invasive ventilation (those on bilevel ventilation or high flow nasal cannula) as a separate subgroup of interest.

When making the recommendation for treatment with remdesivir, the GDG carefully considered the credibility of subgroup findings based on severity of disease, where remdesivir demonstrated a possible survival benefit in patients with severe covid-19, while possibly having no impact on mortality in patients with critical covid-19. The GDG used the ICEMAN tool to assess the credibility of subgroup effects, and ultimately decided the credibility of the observed subgroup finding based on severity of illness was moderate, therefore warranting separate recommendations for each, while recognising residual uncertainties (see MAGICapp for full details regarding ICEMAN assessments).

Balance of benefits and harms-There was low certainty evidence suggesting remdesivir possibly reduces mortality, and moderate certainty evidence suggesting probable reduction in need for mechanical ventilation with probably little or no impact on time to symptom improvement. The drug is well tolerated, and adverse events are rare.

Values and preferences-The GDG inferred that the majority of well informed patients with severe covid-19 would choose to receive remdesivir due to the possible reduction in mortality and need for 
mechanical ventilation and the safety of the drug. The GDG anticipated little variation in values and preferences between patients for this intervention.

Applicability-Insufficient evidence exists to inform a recommendation around use in children. Decisions regarding its use in pregnant or breastfeeding women should, in the absence of trials enrolling such participants, be made between the pregnant person and their healthcare provider while discussing whether the potential benefit justifies the potential risk to the mother and fetus. See MAGICapp for additional guidance.

Practical issues-Remdesivir is administered as one intravenous infusion daily over 10 consecutive days. The recommended dose is $200 \mathrm{mg}$ intravenously on day 1 , followed by $100 \mathrm{mg}$ intravenously on days 2 to 10. Shorter regimens of five days are described in the smaller trials, and local practices may vary. Administration should be as early as possible in the time course of the disease. Patients with severe liver or kidney disease warrant additional caution. See MAGICapp for additional guidance.

Resource implications, acceptability, feasibility, equity, and human rights-Given the intravenous administration of remdesivir daily over 10 days, this is more easily done for hospitalised patients with severe disease, as opposed to the outpatient setting. Obstacles to access in low and middle income countries due to cost, feasibility, and availability are of concern (see box 3 for more details).

\section{Recommendation 2: For patients with critical covid-19, we suggest not to use remdesivir (weak or conditional recommendation).}

\section{Understanding the recommendation}

When moving from evidence to the conditional recommendation not to use remdesivir in patients with critical covid-19, the GDG emphasised the lack of benefit on survival or other patient-important outcomes as demonstrated in the subgroup analysis judged to be of moderate credibility. The GDG recognised there is ongoing uncertainty, and there may still be a subset of patients who would benefit (for example, immunocompromised, persistent viraemia), but there is insufficient evidence to make recommendations specific to these subsets of critical patients.

The GDG did not anticipate important variability in patient values and preferences, although the low certainty of evidence and ongoing uncertainty in effect contributed to the conditional recommendation. There was insufficient trial level data to examine subgroups based on age, or to consider patients requiring non-invasive ventilation (those on bilevel ventilation or high flow nasal cannula) as a separate subgroup of interest.

Balance of benefits and harms-Low certainty evidence suggests remdesivir possibly has little or no effect on mortality and need for mechanical ventilation, and an uncertain effect on time to symptom improvement. The drug is well tolerated, and adverse events are rare.

Values and preferences-The GDG inferred that the majority of well informed patients with critical covid-19 would choose not to receive remdesivir due to little or no impact on patient-important outcomes. The GDG anticipated little variation in values and preferences between patients for this intervention.

Applicability, practical issues, resource implications, acceptability, feasibility, equity, and human rights-Similar issues exist as for patients with severe illness. Such considerations are less relevant for patients with critical illness, given the weak or conditional recommendation against use.
Recommendation 3: We suggest treatment with remdesivir for patients with non-severe covid-19, conditional to those at highest risk of hospitalisation (conditional or weak recommendation).

\section{Understanding the recommendation}

The GDG emphasised the benefits of decreased need for hospitalisation, along with little or no serious adverse effects attributable to the drug. Feasibility, costs, access, and complexity of administration were also carefully considered and led to the conditional recommendation for use only in patients at highest risk of hospitalisation.

Balance of benefits and harms-In highest risk patients with non-severe illness, moderate certainty evidence showed that remdesivir probably provides an important reduction in hospital admissions and may have little or no effect on mortality. The impact of remdesivir on mechanical ventilation and time to symptom resolution is very uncertain. Treatment probably does not increase the likelihood of serious adverse effects leading to drug discontinuation.

Values and preferences-The GDG inferred that almost all well informed patients with a low risk of hospitalisation would decline remdesivir, and only those at highest risk would choose to receive treatment.

Applicability-Only one included trial enrolled children (aged $\geq 12$ years) with small numbers included; the applicability of this recommendation to children therefore remains uncertain. In the absence of trial data for children aged $<12$ years with weight $<40$ $\mathrm{kg}$, the use of remdesivir in these children is not recommended. Uncertainty also remains with regard to administration of remdesivir to pregnant or lactating women. The decision regarding use should be made between the pregnant individual and their healthcare provider while discussing whether the potential benefit justifies the potential risk to the mother and fetus.

The GDG also had concerns regarding whether the drug would retain efficacy against emerging variants of concern, such as omicron BA-1 or BA-2. Surveillance is needed for SARS-CoV-2 strains with reduced susceptibility to remdesivir, and further research is needed to examine the role of combination therapy in severely immunocompromised patients. In the absence of further data, the GDG did not have reason to believe the activity against known variants would be diminished.

Practical issues-Remdesivir is administered via intravenous infusion as a three-day regimen, in keeping with the large trials informing the recommendation; $200 \mathrm{mg}$ is administered intravenously on day 1 , followed by $100 \mathrm{mg}$ given intravenously on days 2 and 3. Administration should be as early as possible in the course of the disease, with monitoring for allergic, infusion related, or other adverse outcomes. In the included studies, remdesivir was administered within seven days of disease onset. Additional considerations regarding practical issues are summarised in MAGICapp.

Resource implications, acceptability, feasibility, equity, and human rights-The infusion schedule represents a feasibility challenge in the outpatient settings. Furthermore, remdesivir is unlikely to be available for all individuals who, given the option, would choose to receive the treatment. See box 3 for concerns about costs, availability, and health inequities. 
Janus kinase (JAK) inhibitors (Update 11, published 16 September 2022)

\section{Overview}

JAK inhibitors inhibit intracellular signalling in response to numerous interleukins, interferons, colony stimulating factors, and hormones. As a consequence, they interfere with many cellular responses, including antiviral responses, angiotensin-converting enzyme 2 (ACE2) expression, T cell function and differentiation, and macrophage activation. Baricitinib, ruxolitinib, and tofacitinib are three of at least nine JAK inhibitors. Their inherent differences, as well as variation in dosing and administration and pharmacokinetics, limit class-wide recommendations, and the GDG decided to make separate recommendations for individual drugs.

The initial strong recommendation concerning baricitinib for patients with severe or critical covid-19 was updated by the GDG in the twelfth version of the living guideline. This followed the availability of new clinical trial evidence for baricitinib administered in combination with corticosteroids and IL- 6 receptor blockers suggesting that the incremental survival benefit afforded by baricitinib exists even among patients also treated with corticosteroids and IL- 6 receptor blockers. ${ }^{16}$

Evidence-For patients with covid-19, data were derived from four trials that enrolled 10815 inpatients for baricitinib, two trials that enrolled 475 inpatients for ruxolitinib, and one trial that enrolled 289 inpatients for tofacitinib. See MAGICapp for detailed description of the mechanism of action, evidence, and subgroup analyses underpinning the recommendation (https://app.magicapp.org/\#/guideline/nBkO1E/rec/E5AOaN).

Recommendation 1: We recommend treatment with baricitinib for patients with severe or critical covid-19 (strong recommendation).

\section{Understanding the recommendation}

In the twelfth iteration, the GDG confirmed the existing strong recommendation to use baricitinib in patients with severe or critical covid-19. This update was based on additional data from 8156 patients enrolled in the RECOVERY trial, which confirmed a survival benefit (now high certainty evidence) and other benefits, with little or no serious adverse events, of a drug that may be administered easily. ${ }^{16}$ The GDG acknowledged that some serious adverse events, such as fungal infections, may not have been accurately captured during the relatively short follow-up period in the included trials. Because of different mechanisms of action, the GDG considered baricitinib separately from other JAK inhibitors.

Costs and access remain important considerations, and the GDG recognises that this recommendation could exacerbate health inequities. This strong recommendation further strengthens the impetus to address these concerns and maximise access across regions and countries. The GDG did not anticipate important variability in patient values and preferences, and judged that other contextual factors would not alter the recommendation.

The GDG had previously made a strong recommendation for use of IL-6 receptor blockers (tocilizumab and sarilumab) or baricitinib as alternative agents administered in addition to corticosteroids for patients with severe or critical covid-19. The GDG had elected to refrain from recommending the combination of these three immunosuppressive drugs until clear evidence of incremental benefit emerged. The RECOVERY trial has now provided this evidence, demonstrating that combining corticosteroids, IL-6 receptor blockers, and baricitinib provides incremental survival benefit. ${ }^{16}$ In RECOVERY, 2659 patients received baricitinib along with corticosteroids and IL-6 receptor blockers. The effect of baricitinib in this subgroup was consistent with the beneficial effect of baricitinib in patients who were not treated with IL-6 receptor blockers. ${ }^{16}$

Although these three immunosuppressive drugs are recommended and may be administered jointly, the panel anticipated that there would be situations where clinicians may opt for less aggressive immunosuppressive therapy or choose to combine medications in a stepwise fashion in patients who are deteriorating. However, since the drugs have not undergone direct comparisons, the GDG felt that clinicians should choose between baricitinib and IL-6 receptor blockers on the basis of experience and comfort using the drugs, local institutional policies, route of administration (baricitinib is oral; IL-6 receptor blockers are intravenous), and cost.

Balance of benefits and harms -In patients with severe or critical illness, baricitinib reduces mortality (high certainty), and probably reduces duration of mechanical ventilation and hospital length of stay (both moderate certainty). Treatment probably results in little or no increase in serious adverse events leading to drug

discontinuation (moderate certainty). Some serious adverse events such as severe infections which may arise from immunosuppressive therapy like baricitinib may not have been accurately captured during the relatively short follow-up in the included trials. This risk may vary in different parts of the world according to the local prevalence of infections such as tuberculosis. This risk may also be less pertinent, given the short course of baricitinib used for the treatment of covid-19.

Subgroup analyses were undertaken for JAK inhibitors as a class (rather than on individual drugs) and revealed no evidence of a subgroup effect on relative risk in younger ( $<70$ years old) versus older patients, those with critical versus severe covid-19, those receiving or not receiving corticosteroids at baseline, and those receiving or not receiving remdesivir or IL-6 blockers at baseline.

Values and preferences-The GDG inferred that almost all well informed patients with severe or critical covid-19 would choose to receive baricitinib due to the likely reduction in mortality, and moderate certainty evidence of little or no increase in serious adverse events. The GDG anticipated little variation in values and preferences between patients for this intervention.

Applicability-None of the included RCTs for baricitinib enrolled children, or pregnant or lactating women; therefore, the applicability of this recommendation to these groups remains uncertain. The GDG did not have reason to believe that patients in these groups with covid-19 would respond differently; decisions regarding the use of JAK inhibitors in these groups should be guided by discussion between the individual and their healthcare provider.

Practical issues-Baricitinib is administered orally once daily as tablets; it can be crushed, dispersed in water, or given via a nasogastric tube. Based on trials informing the recommendation, the recommended dose is $4 \mathrm{mg}$ daily orally in adults with normal renal function for a duration of 14 days or until hospital discharge, whichever is first. The optimal duration of treatment is unknown.

Dose adjustments may be needed for patients with leucopenia, renal impairment, or hepatic impairment, all of which should be monitored during treatment, and for patients taking strong organic anion transporter $3\left(\mathrm{OAT}_{3}\right)$ inhibitors such as probenecid, where drug interactions warrant dose reductions.

Baricitinib, like IL- 6 receptor blockers, should be initiated at the same time as systemic corticosteroids; there are currently no data 
to suggest that specific timing during hospitalisation or the course of illness is beneficial.

See MAGICapp for more information regarding practical issues.

Resource implications, feasibility, equity, and human

rights-Compared with some other candidate treatments for covid-19, baricitinib is expensive. The recommendation does not take into account cost effectiveness. See box 3 for related considerations. As baricitinib is administered orally once daily, hospitalised patients should find it easy to accept this treatment.

\section{Recommendation 2: We suggest not to use ruxolitinib or tofacitinib for patients with severe or critical covid-19 (conditional or weak recommendation).}

\section{Understanding the recommendation}

Low to very low certainty evidence for mortality and duration of mechanical ventilation and a possible increase in serious adverse events, particularly for tofacitinib, drove the weak recommendation not to use ruxolitinib or tofacitinib in patients with severe or critical covid-19. Clinicians should consider using ruxolitinib or tofacitinib only if neither baricitinib nor IL-6 receptor blockers (tocilizumab or sarilumab) are available. The GDG emphasised the need for more trial evidence to better inform the recommendations; this is anticipated through ongoing trials for these JAK inhibitors.

Benefits and harms-Low to very low certainty evidence from small trials failed to demonstrate benefits for mortality or duration of mechanical ventilation, and suggested tofacitinib may increase adverse events leading to drug discontinuation. When more evidence is available, the GDG acknowledged that these drugs may prove to have similar benefits as baricitinib.

Values and preferences-Most well informed patients would decline ruxolitinib or tofacitinib. However, a minority might choose to receive one or the other drug if neither baricitinib nor IL-6 receptor blockers are available, given that the possibility of benefit has not been excluded and a class effect of JAK inhibitors might exist.

Applicability-None of the included RCTs enrolled children; therefore, the applicability of this recommendation to children remains uncertain. Uncertainty also remains with regards to the administration of ruxolitinib or tofacitinib to pregnant or lactating women.

Practical issues-Both drugs are administered orally twice daily as tablets and can be dispersed in water or administered via nasogastric tube.

The GDG referred to treatment regimens in the included trials, available via MAGICapp, in the absence of other available information. If ruxolitinib or tofacitinib is administered, like with IL-6 receptor blockers, it should be given with systemic corticosteroids; specific timing during hospitalisation or in the context of the course of illness is not specified.

Resource implications, equity, and human rights-Efforts to ensure access to drugs should focus on those that are currently recommended.

Specific uncertainties, emerging evidence, and future research (for all JAK inhibitors)

- Safety and efficacy of combination therapy of baricitinib with corticosteroids and IL-6 receptor blockers on longer term outcomes
- Safety and efficacy in areas where certain infections (such as HIV infection, tuberculosis, and some fungal infections) are endemic

- Impact of tofacitinib and ruxolitinib relative to that of baricitinib.

Sotrovimab (neutralising monoclonal antibodies) (Update 12, published 13 January 2023)

\section{Overview}

Sotrovimab is a single human monoclonal antibody that binds to a conserved epitope in the SARS-CoV-2 spike protein, preventing the virus from entering cells.

Update-An updated recommendation concerning sotrovimab for patients with non-severe covid-19 was published in the twelfth version of the living guideline. Previously, a conditional recommendation was provided for use in patients with non-severe covid-19 at highest risk of hospitalisation. Following the emergence of the currently circulating SARS-CoV-2 variants (such as omicron) and subvariants now dominating covid-19 worldwide, and availability of evidence showing sotrovimab has very diminished in vitro neutralisation activity to these subvariants, the GDG made a strong recommendation against the use of sotrovimab.

In this thirteenth iteration of the guideline, updated data showing in vitro neutralisation activity is diminished for currently circulating SARS-CoV-2 variants (that is, omicron) became available, confirming the previous strong recommendation against use of sotrovimab for patients with non-severe covid-19. See MAGICapp for detailed description of the mechanism of action, detailed in vitroneutralisation activity data, and rationale underpinning the recommendation (https://app.magicapp.org/\#/guide-

line/nBkO1E/rec/LA69PM).

Recommendation: We recommend not to use sotrovimab for patients with non-severe covid-19 (strong recommendation).

\section{Understanding the recommendation}

Although previous clinical trial evidence available via the living network meta-analysis (LNMA) remains accurate, ${ }^{7}$ the panel concluded that it is no longer applicable to covid-19 caused by the SARS-CoV-2 variants and subvariants that are currently circulating globally. The panel surmised that the likelihood of covid-19 caused by former variants was extremely low, and that, accordingly, evidence of sotrovimab's clinical effectiveness for covid-19 was inexistent.

The GDG reviewed additional in vitro neutralisation data pertaining to new variants and subvariants that was made available after the twelfth iteration of the guideline. This incremental evidence supports the change in recommendation, and strengthens the GDG's confidence that the strong recommendation not to use sotrovimab (and casirivimab-imdevimab) is applicable to the current SARS-CoV-2 ecology. More information on the interpretation of the results of in vitro neutralisation data can be found in MAGICapp and in correspondence published in the ancet $^{17}$ Of note, the GDG applied the same rationale to the recommendation for the monoclonal antibody combination casirivimab-imdevimab.

The GDG agreed that large, high quality clinical trials generally provide the best evidence of clinical effectiveness for therapeutic interventions. The GDG also continues to base its recommendations strictly on critically important outcomes. From the perspective of clinical guidelines, mechanistic studies and surrogate outcomes are useful to identify candidate therapies for clinical trials but are of no use in confirming clinical effectiveness. The panel concluded 
that the emerging evidence demonstrating the reduced neutralisation of current variants by sotrovimab in vitro would likely have justified not launching clinical trials and now renders the results of previous trials inapplicable. In vitro assays were deemed sufficient to rule out a clinical effect. Notwithstanding, proof of potent in vitro neutralisation would not be sufficient to confirm clinical effectiveness. Therefore, the GDG will only consider making recommendations for new monoclonal antibodies once they have been rigorously evaluated in clinical trials.

Balance of benefits and harms-There was consensus among the panel that it is highly unlikely that the clinical effectiveness of sotrovimab would persist in the absence of adequate in vitro neutralisation of the circulating variants and subvariants. Accordingly, the panel concluded that the evidence upon which hinged the previous recommendation was no longer applicable.

Values and preferences-The GDG inferred that, in the absence of compelling evidence of clinical effectiveness for the currently circulating SARS-CoV-2 variants and subvariants, almost all well informed patients would not choose to receive sotrovimab.

Applicability-Given the updated recommendation against treatment, issues pertaining to applicability were felt to be less relevant.

Practical issues-Given the updated recommendation against treatment, related practical issues were felt to be less relevant.

Resource implications, equity, human rights, acceptability, and feasibility-The strong recommendation against the use of sotrovimab is further supported by their challenges with availability and feasibility, such as limited production, intravenous administration, and requirement for expertise to offer such treatment while oral antiviral therapies are available.

\section{Specific uncertainties, emerging evidence, and future research}

- Efficacy and safety for severe or critical seronegative covid-19 patients

Casirivimab-imdevimab (neutralising monoclonal antibodies) (Update 12, published 13 January 2023)

\section{Overview}

Casirivimab and imdevimab are two fully human antibodies (REGN10933 and REGN10987) that bind to the SARS-CoV-2 spike protein and have demonstrated antiviral activity in animal models. It has been postulated that administration of a combination of casirivimab and imdevimab might have differential effects in patients who have produced their own anti-SARS-CoV-2 spike protein antibodies (hereafter seropositive) compared with those who have not (hereafter seronegative); it was hypothesised that effects might be larger for, or restricted to, seronegative individuals who have not yet mounted an effective natural antibody response.

\section{Update}

An updated recommendation concerning the neutralising antibodies casirivimab-imdevimab for patients with covid-19 was published in the twelfth version of the WHO living guideline. Previously, a conditional recommendation was provided for patients with non-severe covid-19 at highest risk of hospitalisation, and for patients with severe or critical illness with seronegative status. Following the emergence of the currently circulating SARS-CoV-2 variants and subvariants (such as omicron) now dominating worldwide, and availability of in vitro data showing lack of or diminished neutralisation activity, the GDG made a strong recommendation against the use of casirivimab-imdevimab for all patients with covid-19.

In this thirteenth iteration of the guideline, updated data showing in vitro neutralisation activity is absent or diminished for currently circulating SARS-CoV-2 variants (that is, omicron) and subvariants became available, further affirming the previous strong recommendation against use of casirivimab-imdevimab. See MAGICapp for detailed description of the mechanism of action, detailed in vitro neutralisation activity data, and rationale underpinning the recommendation (https://app.magicapp.org/\#/guideline/nBkO1E/section/LG5NRE).

Recommendation: We recommend not to use casirivimab-imdevimab for patients with covid-19, regardless of illness severity (strong recommendation).

\section{Understanding the recommendation}

Although previous clinical trial evidence available via the LNMA remains accurate, ${ }^{7}$ the GDG concluded that it is no longer applicable to covid-19 caused by the SARS-CoV-2 variants and subvariants that are currently circulating globally. The panel surmised that the likelihood of covid-19 caused by former variants was extremely low and that, accordingly, evidence of casirivimab-imdevimab clinical effectiveness for covid-19 was inexistent.

The GDG reviewed additional in vitro neutralisation data pertaining to new variants and subvariants that was made available after the twelfth iteration of the guideline. This incremental evidence supports the change in recommendation, and strengthens the GDG's confidence that the strong recommendation not to use casirivimab-imdevimab (and sotrovimab) is applicable to the current SARS-CoV-2 ecology. More information on the interpretation of the results of in vitro neutralisation data can be found in MAGICapp and in a letter to the editor published in the Lancet. ${ }^{17}$ Of note, the panel applied the same rationale to the recommendation for sotrovimab.

The GDG agreed that large, high quality clinical trials generally provide the best evidence of clinical effectiveness for therapeutic interventions. The GDG also continues to base its recommendations strictly on predefined patient-important outcomes. From the perspective of clinical practice guidelines, mechanistic studies and surrogate outcomes are useful to identify candidate therapies for clinical trials but are of no use in the evaluation of clinical effectiveness. The panel concluded that the emerging evidence demonstrating that casirivimab-imdevimab did not comparatively neutralise current variants in vitro would have justified not launching clinical trials and now renders the results of previous trials inapplicable. In vitro assays were deemed sufficient to rule out a clinical effect. Notwithstanding, proof of potent in vitro neutralisation would not be sufficient to confirm clinical effectiveness. Therefore, the GDG will only consider making recommendations for new monoclonal antibodies once they have been rigorously evaluated in clinical trials.

Balance of benefits and harms-There was consensus among the panel that it is highly unlikely that the clinical effectiveness of casirivimab-imdevimab would persist in the absence of adequate in vitro neutralisation of the circulating variants and subvariants. Accordingly, the panel concluded that the evidence upon which hinged the previous recommendations was no longer applicable.

Values and preferences-The GDG inferred that, in the absence of compelling evidence of clinical effectiveness for the currently circulating SARS-CoV-2 variants and subvariants, almost all well 
informed patients would not choose to receive casirivimab-imdevimab.

Applicability-Given the updated recommendation against treatment, issues pertaining to applicability were felt to be less relevant.

Practical issues-Given the updated recommendation against treatment, related practical issues were felt to be less relevant.

Resource implications, acceptability, feasibility, equity, and human rights-The strong recommendation against the use of casirivimab-imdevimab is further supported by their challenges with availability and feasibility, such as limited production, intravenous administration and requirement for expertise to offer such treatment while oral options are available.

\section{Specific uncertainties, emerging evidence, and future research}

No specific uncertainties: see uncertainties section in "How to use this guideline" (above).

Fluvoxamine (Update 10, published 14 July 2022)

\section{Overview}

Fluvoxamine is a selective serotonin reuptake inhibitor (SSRI) approved as an antidepressant. It increases concentrations of serotonin in the synaptic cleft. Indirect evidence from non-covid-19 disease models have suggested possible anti-inflammatory properties, and mechanistic studies have suggested host-directed antiviral properties. There are currently no published preclinical studies that directly demonstrate a mechanism in covid-19.

Evidence-For patients with non-severe covid-19, data were derived from three trials that enrolled 2208 non-hospitalised patients. See MAGICapp for detailed description of the mechanism of action, evidence, and subgroup analyses underpinning the recommendation (https://app.magicapp.org/\#/guideline/nBkO1E/rec/jmYxpV).

Recommendation 1: For patients with non-severe covid-19, we recommend not to use fluvoxamine, except in the context of a clinical trial (recommended only in a research setting).

\section{Understanding the recommendation}

Insufficient evidence of benefit, coupled with a lack of a clear mechanism of action and known drug interactions, drove the recommendation against use in clinical care.

Balance of benefits and harms-There was low to moderate certainty evidence suggesting little or no effect on hospitalisation, mortality, or mechanical ventilation, and an absence of reliable data on serious adverse effects attributable to the drug. Fluvoxamine is known for substantial pharmacological interactions. In the largest trial, markedly more patients discontinued treatment in the fluvoxamine group than in the placebo group. Acknowledging that its evaluation of the certainty of the evidence may differ from other published meta-analyses, GDG members pointed out that early stopping due to apparent benefit may have biased the results of the largest trial. They argued that, although the stopping rules were pre-specified, the decision to stop the trial was based on the effect estimate on a composite outcome of questionable importance; meanwhile the number of important events was lower and vulnerable to bias. The GDG also raised concerns regarding the uncertain applicability of this trial conducted in a single country.

Values and preferences-The GDG inferred that almost all well informed patients would choose not to receive fluvoxamine therapy for covid-19 based on available evidence. The GDG did not believe that other considerations, such as feasibility, acceptability, equity, and cost, would affect this specific recommendation. Specifically, the GDG did not consider the potential role of fluvoxamine as an antidepressant for this guideline of medications for covid-19.

Applicability-None of the included studies enrolled children, and the applicability of this recommendation to children is therefore uncertain. However, the GDG did not see a reason to assume that children with covid-19 would respond any differently to treatment with fluvoxamine.

Practical issues-The GDG made a recommendation against using fluvoxamine for treatment of patients with covid-19 outside the setting of a clinical trial, and therefore practical considerations are less relevant for this drug.

Resource implications, acceptability, feasibility, equity, and human rights-Fluvoxamine is relatively inexpensive compared with other drugs used for covid-19, and widely available, including in low income settings. Its use would risk diverting attention and resources away from interventions that are more likely to provide a benefit.

Specific uncertainties, emerging evidence, and future research-The current evidence does not justify using fluvoxamine to treat covid-19. However, the recommendation does not imply that fluvoxamine is proven to be ineffective. Decisions to further investigate the effects of fluvoxamine for covid-19 will likely hinge on how stakeholders perceive the opportunity cost of investigating the effects of fluvoxamine over other candidate therapies. The GDG discussions highlighted the following knowledge gaps:

- What are the effects of fluvoxamine in patients who have a combination of non-severe covid-19 that is at risk of deteriorating and significant symptoms of anxiety?

- What are the side effects of fluvoxamine therapy in patients with non-severe covid-19 at risk of deteriorating?

- What proportion of patients with non-severe covid-19 at risk of deteriorating would be ineligible due to risk of pharmacological interactions?

The GDG surmised that, in the future, investigators would have to carefully consider whether fluvoxamine could still be compared with placebo or no treatment given that effective treatments are available. If the rationale to further investigate fluvoxamine is its advantageous cost and availability, non-inferiority designs may be considered.

\section{Colchicine (Update 10, published 14 July 2022)}

\section{Overview}

Colchicine is an anti-inflammatory drug used to treat gout, recurrent pericarditis, familial Mediterranean fever, and other inflammatory conditions. Proposed mechanisms for its anti-inflammatory effect include a reduction in neutrophil chemotaxis, inflammasome signalling inhibition, and decreased production of cytokines such as interleukin $1 \mathrm{~b}$. No published animal models evaluating colchicine in SARS-CoV-2 infection are published at the time of guideline publication.

Evidence-For patients with non-severe covid-19, data were derived from 10 trials that enrolled 17914 non-hospitalised patients. See MAGICapp for detailed description of the mechanism of action, evidence, and subgroup analyses underpinning the recommendation 
Recommendation 1: For patients with non-severe covid-19, we recommend against treatment with colchicine (strong recommendation).

\section{Understanding the recommendation}

The lack of benefits on hospitalisations, mortality, and mechanical ventilation, combined with possible harms and toxicity, drove the strong recommendation against the use of colchicine in patients with non-severe covid-19.

Balance of benefits and harms-In patients with non-severe covid-19, colchicine has little or no impact on mortality or mechanical ventilation (moderate certainty). It is unclear whether it affects hospitalisations or adverse effects leading to drug discontinuation. The GDG discussed the risk of drug interactions and colchicine's narrow therapeutic window, particularly in patients with or at risk of hepatic and renal failure. Colchicine toxicity can be severe and sometimes fatal. The planned subgroup analyses for colchicine versus standard care did not show different relative effects for disease severity or age (children, adults, older adults), with no data reported from illness onset.

Values and preferences-The GDG inferred that almost all well informed patients would choose not to receive colchicine.

Applicability-The applicability of this recommendation to children is currently uncertain because none of the included studies enrolled children. However, the GDG did not consider that children with covid-19 would respond any differently to treatment with colchicine.

Practical issues-The GDG made a strong recommendation against using colchicine for treatment of patients with non-severe covid-19, and therefore practical considerations are less relevant.

Resource implications, acceptability, feasibility, equity, and human rights-These considerations did not affect this specific recommendation. Although colchicine is relatively inexpensive compared with other drugs used for covid-19, and widely available, including in low income settings, the evidence does not justify the use of colchicine for non-severe covid-19 anywhere. Although the cost of colchicine may be low, the GDG raised concerns regarding the risk of diverting attention and resources away from interventions that are more likely to provide a benefit.

Specific uncertainties, emerging evidence, and future research-The GDG considered it unlikely that future studies would identify subgroups of patients who would benefit from colchicine.

\section{Molnupiravir (Update 8, published 01 March 2022)}

\section{Overview}

Molnupiravir is an antiviral administered orally. It was re-purposed as an antiviral for covid-19 because it inhibits replication of SARS-CoV-2 with an in vitro potency broadly similar to remdesivir. ${ }^{18} 19$ This inhibitory effect has been shown in animal studies, both at higher and lower doses, with possibly greater efficacy when combined with favipiravir (compared with either drug alone). ${ }^{20}-22$ The drug is active against alpha and beta variants in vivo based on studies in hamsters and human cell models, and delta and omicron variants in vitro (no data in vivo). ${ }^{23-25}$ In vitro and animal studies have suggested the possibility of carcinogenesis; no human data with long term follow-up are available regarding this. There is also residual uncertainty regarding other long term harms; the efficacy of the drug against variants, particularly those with higher replication or transmission rates; the possibility of a selective pressure for resistant mutations at an individual level, with the potential to spread at a population level; and the emergence of new variants related to random mutagenesis arising from molnupiravir's mechanism of action. These issues are comprehensively described in the full version of the guideline (see box 1).

Evidence-For patients with non-severe covid-19, data were derived from six trials that enrolled 4827 patients, of which the LNMA team had access to data for 4796 patients. See MAGICapp for detailed description of the mechanism of action, evidence, and subgroup analyses underpinning the recommendation (https://app.magicapp.org/\#/guideline/nBkO1E/rec/E85WNb).

Recommendation 1: We suggest treatment with molnupiravir for patients with non-severe covid-19, conditional to those at highest risk of hospitalisation (conditional or weak recommendation).

\section{Understanding the recommendation}

Only a minority of patients who are at highest risk are likely to achieve sufficient benefit. Especially given the safety concerns related to molnupiravir, the WHO and the GDG recognise the need to mitigate risks, both for individual patients and at the population level.

Balance of benefits and harms-Molnupiravir probably provides benefits on admission to hospital and time to symptom resolution (both moderate certainty) and may have a small effect on mortality (low certainty), without an increase in short term adverse effects (high certainty). Absolute benefits depend on the prognosis of the individual patient. The GDG also considered potential long term harms of molnupiravir, including risk of malignancy based on preclinical data (very low certainty), in what they ultimately considered to be a close balance between benefits and harms.

Values and preferences-The GDG inferred that almost all well informed patients with a low risk of hospitalisation would decline molnupiravir, and only those at highest risk would choose to receive treatment.

Applicability-

- Children. Due to evidence of impact on growth plate thickness and decreased bone formation in some animal studies, molnupiravir should not be used in children.

- Pregnancy, breastfeeding, and conception. Since molnupiravir elicited embryo-fetal lethality and teratogenicity in offspring when given to pregnant animals, it should not be used in pregnant or breastfeeding women. If pregnancy status is unclear, one should perform a pregnancy test before starting molnupiravir treatment. Women and people who can get pregnant should be counselled regarding reducing the risk of conception (such as using birth control) during treatment and for at least four days after the last dose of molnupiravir.

- Uncertainty remains regarding consequences to children conceived by fathers receiving or having recently received molnupiravir, and whether spermatogenesis may be especially prone to mutagenic effects. Men who might father a child should use birth control during treatment and for at least three months after the last dose of molnupiravir.

- Mitigation strategies at the population level include active sequence monitoring of SARS-CoV-2 detected in clinical respiratory samples for patients receiving therapy and active pharmacovigilance programmes.

Practical issues-As per large trials informing the recommendation, molnupiravir is dosed as $800 \mathrm{mg}$ orally every 12 hours for five days. 
Administration should be as early as possible in the course of the disease. In the included studies, molnupiravir was administered within five days of symptom onset.

Resource implications, feasibility, equity, and human rights-Molnupiravir is unlikely to be available for all individuals who, given the option, would choose to receive the treatment.

\section{Specific uncertainties, emerging evidence, and future research}

- Need for clinical data to investigate safety and applicability concerns (including in children, lactating or pregnant women, and men; and long term impact on mutagenesis and cancer risk).

\section{Convalescent plasma (Update 6, published 6 December 2021)}

\section{Overview}

Treatment with convalescent plasma involves the transfer of endogenously produced neutralising antibodies present within the plasma from previously infected and recovered patients into patients with active infection. The concentrations (titre) of neutralising antibodies present within convalescent plasma are highly variable between donors, and various methodologies to measure antibody levels are available.

Evidence-Data were derived from 16 trials enrolling 16236 patients across illness severities, of which four RCTs with 1602 patients informed estimates for outcomes in non-severe illness. See MAGICapp for detailed description of the mechanism of action, evidence, and subgroup analyses underpinning the recommendation (https://app.magicapp.org/\#/guideline/nBkO1E/section/LG5NRE).

Recommendation 1: We recommend not to use convalescent plasma for patients with non-severe covid-19 (strong recommendation).

\section{Understanding the recommendation}

The GDG noted that, although not demonstrated in the evidence summary, there remains a potential for harms with blood product transfusion. Most importantly, given there was no benefit demonstrated for any of the critical or important outcomes for non-severe covid-19, the GDG did not see any justification for the resources (including time and cost) that would be associated with administration of convalescent plasma.

Balance of benefits and harms-In patients with non-severe illness, convalescent plasma does not have an important impact on mortality (high certainty). Convalescent plasma probably does not affect mechanical ventilation (moderate certainty). There were no data evaluating the risk of hospitalisation with convalescent plasma; the impact is therefore very uncertain. Convalescent plasma probably does not result in important increases in risks of transfusion-related acute lung injury, transfusion-associated circulatory overload (both moderate certainty), or allergic reactions (low certainty).

Values and preferences-Applying the agreed values and preferences, the GDG inferred that almost all well informed patients with non-severe covid-19 would choose against receiving convalescent plasma.

Acceptability and applicability-Although blood transfusion is acceptable to most, there is a subset of the population who will not accept allogenic blood transfusions. There are also regulatory challenges in most jurisdictions related to blood product transfusions. The included RCTs enrolled non-pregnant women and men. The GDG did not have reason to believe that children or pregnant women with covid-19 would respond any differently to treatment with convalescent plasma; the GDG therefore inferred that children and pregnant women should not receive the intervention either.

Practical issues-Issues include, though are not limited to, the identification and recruitment of potential donors, collection of plasma, storage and distribution of plasma, and infusion of convalescent plasma into recipients.

Resource implications, feasibility, equity, and human rights-The GDG noted that convalescent plasma use is associated with significant resource requirements, including identification of potential donors, testing of donors to ensure adequate titres of anti-SARS-CoV-2 antibodies, collection of donor plasma, storage of plasma, transportation of plasma to recipient location, and administration of plasma. These resources and feasibility issues are compounded for those with non-severe illness, who are most often outpatients. Also, this process is costly and time consuming. Given the number of patients with non-severe illness and the low event rate in this subgroup of patients, mobilising the use of convalescent plasma on a large scale would be of questionable feasibility.

Recommendation 2: We recommend not to use convalescent plasma for patients with severe or critical covid-19, except in the context of a clinical trial (recommended only in research settings).

\section{Understanding the recommendation}

Given relative benefits and harms, the GDG agreed further research addressing these patient-important outcomes would be valuable for patients with severe or critical illness. A recommendation to use a drug only in the setting of clinical trials is appropriate when there is low certainty evidence, and future research has potential to reduce uncertainty about the effects of the intervention, and for doing so at a reasonable cost.

Balance of benefits and harms -In patients with severe or critical covid-19, convalescent plasma may not result in an important impact on mortality, mechanical ventilation, time to symptom improvement, length of hospital stay, or ventilator-free days (all low or very low certainty). Convalescent plasma probably does not result in important increases in risks of transfusion-related acute lung injury, transfusion-associated circulatory overload (both moderate certainty), or allergic reactions (low certainty). However, there is always potential for harms with blood product transfusions.

Values and preferences-Applying the agreed values and preferences, the GDG inferred that almost all well informed patients would choose against receiving convalescent plasma outside the research setting.

Specific uncertainties, emerging evidence, and future research

- Effects of high titre convalescent plasma on mortality and other patient-important outcomes.

- Effects in patients with seronegative antibody status.

Interleukin-6 (IL-6) receptor blockers (Update 4, published 6 July 2021)

\section{Overview}

IL-6 receptor blockers tocilizumab and sarilumab are monoclonal antibodies approved for use in rheumatoid arthritis. Elevated IL-6 concentrations are associated with severe outcomes in covid-19, including respiratory failure and death. IL-6 receptor blockers antagonise membrane-bound and soluble forms of the IL- 6 receptor, blocking the cytokine's activation and regulation of the immune response to infection. 
WHO has made a strong recommendation for JAK inhibitors, specifically baricitinib, in patients with severe or critical covid-19. As per guidance updated in the twelfth iteration of the guideline, an IL-6 receptor blocker and baricitinib may be given as combination therapy (see discussion for JAK inhibitors above).

Evidence-In addition to the linked network meta-analysis, this recommendation was also informed by an independent prospective meta-analysis from the WHO Rapid Evidence Appraisal for covid-19 group. ${ }^{26}$ The network meta-analysis included 30 RCTs with 10618 participants, and these data were used by the GDG for all outcomes other than mortality. We used the prospective meta-analysis for mortality because it included additional data that was unpublished at the time. The prospective meta-analysis pooled data from 22 RCTs with 10156 participants. ${ }^{26}$ See MAGICapp for detailed description of the mechanism of action, evidence, and subgroup analyses underpinning the recommendation (https://app.magicapp.org/\#/guideline/nBk01E/section/LG5NRE).

Recommendation: We recommend treatment with IL- 6 receptor blockers (tocilizumab or sarilumab) for patients with severe or critical covid-19 (strong recommendation).

\section{Understanding the recommendation}

Of note, corticosteroids have previously been strongly recommended in patients with severe or critical covid-19, and we recommend that patients meeting these severity criteria should now receive both corticosteroids and IL-6 receptor blockers, possibly with baricitinib as combination therapy (see above).

Balance of benefits and harms - IL- 6 receptor blockers reduce mortality and need for mechanical ventilation (both high certainty), and may reduce durations of mechanical ventilation and hospitalisation (both low certainty).

There was uncertainty about the risk of serious adverse effects (very low certainty). There may be little or no increased risk of bacterial infections. However, the GDG had some concerns that, given the short term follow-up of most trials and the challenges associated with accurately capturing adverse events such as bacterial or fungal infections, that the evidence summary may under-represent the risks of treatment with IL-6 receptor blockers. Furthermore, the trials of IL-6 receptor blockers that inform this recommendation were mostly performed in high-income countries, where the risk of infectious complications may be less than in some other parts of the world; the generalisability of the data on these adverse events is therefore unclear.

Values and preferences-The GDG inferred that almost all well informed patients with severe or critical covid-19 infection would want to receive IL-6 receptor blockers, given the reduction in mortality and mechanical ventilation, despite low certainty around evidence for serious adverse events. A minority of the GDG felt that a significant proportion of patients might decline the intervention due to the uncertainties around harms, and taking into account the small reduction in mortality.

Applicability-None of the included RCTs enrolled children or pregnant women. Although this resulted in uncertain applicability, the GDG did not have reason to believe that children or pregnant women with covid-19 would respond any differently to treatment with IL-6 receptor blockers.

Practical issues-IL-6 receptor blockers require intravenous administration but only require one, or at most two, doses. See MAGICapp for practical information, including considerations if
IL-6 receptor blockers are considered in children and pregnant women.

Resource implications, acceptability, feasibility, equity, and human rights-Compared with other treatments for covid-19, IL-6 receptor blockers are expensive and may be inaccessible. The recommendation does not consider cost effectiveness. Given limited availability of the drug, one may consider the relative effects (odds ratio 0.87 ) for reduction in mortality with IL-6 receptor blockers result in 28 fewer deaths per 1000 patients ( $95 \%$ confidence interval 9 to 47 fewer deaths) in critically ill patients, compared with 12 fewer deaths per 1000 patients ( 4 to 19 fewer deaths) in severely ill patients.

Finally, sarilumab is not indicated for use in children; therefore, there could be a preference for tocilizumab in this subgroup.

Specific uncertainties, emerging evidence, and future research

- Safety data, including nosocomial infections.

- Immunity and the risk of subsequent infection, which may affect the risk of death after 28 days.

- Outcomes by different IL-6 receptor blocker dosing, and optimal timing of drug initiation.

\section{Ivermectin (Update 3, published 31 March 2021)}

\section{Overview}

Ivermectin is an antiparasitic agent that interferes with nerve and muscle function of helminths through binding glutamate-gated chloride channels. The treatment is relatively inexpensive and accessible internationally. We currently lack persuasive evidence of a mechanism of action for ivermectin in covid-19; any observed clinical benefit would be unexplained.

We are aware of a few new, relatively small trials published since our recommendation was made, and that one key trial has since been retracted, given concerns about research fraud. ${ }^{2728}$ However, the updated evidence summary from the living network meta-analysis is consistent with our previous recommendation. This updated evidence summary will be fully considered by the GDG in an upcoming iteration of the guideline.

Evidence-The living systematic review and network meta-analysis pooled data from 16 trials with 2407 participants. Of the included trials, $75 \%$ examined patients with non-severe illness, and $25 \%$ included patients with both severe and non-severe illness. See MAGICapp for detailed description of the mechanism of action, evidence, and subgroup analyses underpinning the recommendation (https://app.magicapp.org/\#/guideline/nBkO1E/section/LG5NRE).

Recommendation: We recommend not to use ivermectin for patients with covid-19, regardless of illness severity, except in the context of a clinical trial (recommended only in research settings).

\section{Understanding the recommendation}

Very low certainty evidence was a critical factor in the recommendation.

Balance of benefits and harms-Certainty of evidence for mortality was deemed very low, despite a point estimate and confidence interval that seemed to suggest benefit with ivermectin; similar judgments were made for other outcomes, including mechanical ventilation, hospital admission, duration of hospitalisation, and viral clearance. 
Ivermectin may have little or no effect on time to clinical improvement (low certainty) and may increase the risk of adverse events leading to drug discontinuation (low certainty). A recommendation to only use a drug in the setting of clinical trials is appropriate when there is very low certainty evidence, and when future research has large potential for reducing uncertainty about the effects of the intervention and at a reasonable cost.

Subgroup analyses indicated no effect modification based on dose. We were unable to examine subgroups based on age or severity of illness due to insufficient trial data. Therefore, we assumed similar effects across all subgroups.

Values and preferences-The GDG inferred that almost all well informed patients would not want to receive ivermectin, given available evidence left a very high degree of uncertainty in effects on critical outcomes and the possibility of harms, such as adverse events associated with treatment.

Applicability-None of the included trials enrolled children or pregnant women; the applicability of the evidence to these subgroups is therefore uncertain, though there is no rationale to suggest they would respond differently.

Resource implications, acceptability, feasibility, equity, and human rights-Although the cost of ivermectin may be low per patient, the GDG raised concerns about diverting attention and resources away from care likely to provide a benefit, such as corticosteroids in patients with severe covid-19, and other supportive care interventions. Also, use of ivermectin for covid-19 would divert supply away from pathologies for which it is clearly indicated, potentially contributing to drug shortages, especially for helminth control and elimination programmes. If corticosteroids are used in the treatment of covid-19, empiric treatment with ivermectin may still be considered in strongyloidiasis-endemic areas, albeit not for treatment of covid-19 itself.

\section{Specific uncertainties, emerging evidence, and future research}

No specific uncertainties: see uncertainties section in "How to use this guideline" (above).

Hydroxychloroquine (Update 2, published 17 December 2020)

Evidence-The recommendation addressing hydroxychloroquine was informed by results from the living network meta-analysis, pooling data from 30 RCTs with 10921 participants. See MAGICapp for detailed description of the evidence and subgroup analyses underpinning the recommendation (https://app.magicapp.org/\#/guideline/nBkO1E/section/j197zj).

Recommendation: We recommend not to use hydroxychloroquine or chloroquine for patients with covid-19, regardless of illness severity (strong recommendation).

\section{Understanding the recommendation}

Balance of benefits and harms-Hydroxychloroquine and chloroquine probably do not reduce mortality or mechanical ventilation (both moderate certainty) and may have no effect on duration of hospitalisation (low certainty). The evidence does not exclude the potential for a small increased risk of death and mechanical ventilation with hydroxychloroquine. The effect on other less important outcomes, including time to symptom resolution, admission to hospital, and viral clearance, remains uncertain.

Hydroxychloroquine may increase the risk of diarrhoea and nausea or vomiting (both low certainty), a finding consistent with evidence from its use in other conditions. Diarrhoea and vomiting may increase the risk of hypovolaemia, hypotension, and acute kidney injury, especially in settings where healthcare resources are limited. Whether and to what degree hydroxychloroquine increases the risk of cardiac toxicity, including life-threatening arrhythmias, when used in patients with covid-19 is uncertain (very low certainty).

Subgroup analyses indicated no effect modification based on severity of illness, age, cumulative dose, or predicted day 3 serum trough concentrations. Therefore, we assumed similar effects in all subgroups.

We also reviewed evidence comparing the use of hydroxychloroquine plus azithromycin versus hydroxychloroquine alone. There was no evidence that the addition of azithromycin modified the effect of hydroxychloroquine for any outcome (very low certainty).

Values and preferences-Applying the agreed values and preferences, the GDG inferred that almost all well informed patients would not want to receive hydroxychloroquine.

Applicability-None of the included trials enrolled children or adolescents; the applicability to this subgroup is therefore uncertain.

Resource implications, feasibility, equity, and human

rights-Hydroxychloroquine and chloroquine are relatively inexpensive compared with other drugs used for covid-19 and are already widely available, including in low-income settings. Although the cost may be low per patient, the GDG raised concerns about diverting attention and resources away from care likely to provide a benefit such as corticosteroids in patients with severe covid-19 and other supportive care interventions.

\section{Specific uncertainties, emerging evidence, and future research}

Although some uncertainty remains, the GDG felt that further research was unlikely to uncover a subgroup of patients who would benefit from hydroxychloroquine on the most important outcomes (mortality, mechanical ventilation) given the consistent results in trials across illness severity and location.

\section{Lopinavir-ritonavir (Update 2, published 17 December 2020)}

Evidence-The recommendation was informed by data from seven RCTs with 7429 participants. See MAGICapp for detailed description of the evidence and subgroup analyses underpinning the recommendation (https://app.magicapp.org/\#/guideline/nBkO1E/section/EgylxL).

Recommendation: We recommend not to use lopinavir-ritonavir for patients with covid-19, regardless of illness severity (strong recommendation).

Balance of benefits and harms - Lopinavir-ritonavir probably has little or no effect on mortality and mechanical ventilation (both moderate certainty); effects on time to clinical improvement and other patient-important outcomes were uncertain (low or very low certainty). Treatment probably increases the risk of diarrhoea and nausea or vomiting (both moderate certainty), a finding consistent with the indirect evidence evaluating its use in patients with HIV infection. Diarrhoea and vomiting may increase the risk of hypovolaemia, hypotension, and acute kidney injury, especially in settings where healthcare resources are limited. There was an uncertain effect on viral clearance and acute kidney injury.

Subgroup analysis indicated no effect modification based on severity of illness or age. As there was no evidence of a statistical subgroup effect, we did not formally evaluate credibility. Although the trials did not report subgroup effects by time from symptom onset, many 
of the trials enrolled patients early in the illness course. The GDG therefore felt that the evidence applies to all patients with covid-19.

Values and preferences-Applying the agreed values and preferences, the GDG inferred that almost all well informed patients would not want to receive lopinavir-ritonavir given that the evidence suggested there was probably no effect on mortality or need for mechanical ventilation and there was a risk of adverse events including diarrhoea and nausea or vomiting.

Resource implications, feasibility, equity, and human rights-Although the cost of lopinavir-ritonavir is not as high as some other investigational drugs for covid-19 and the drug is generally available in most healthcare settings, the GDG raised concerns about opportunity costs and the importance of not drawing attention and resources away from best supportive care or the use of corticosteroids in severe covid-19.

\section{Specific uncertainties, emerging evidence, and future research}

Although some uncertainty remains, the GDG felt that further research was unlikely to uncover a subgroup of patients who would benefit from lopinavir-ritonavir on the most important outcomes (mortality, mechanical ventilation) given the consistent results in trials across illness severity and location.

\section{Systemic corticosteroids (Original publication, published 4 September 2020)}

Evidence-The GDG reviewed evidence from eight RCTs (7184 patients) evaluating systemic corticosteroids versus usual care in treatment of covid-19, seven of which reported mortality data by subgroup of illness severity. Mortality data from one trial, GLUCOCOVID, were not incorporated in the summary of finding for mortality because the mortality outcome data were not available by subgroup. See MAGICapp for detailed description of the evidence and subgroup analyses underpinning the recommendation and practical information on how to administer systemic corticosteroids (https://app.magicapp.org/\#/guideline/nBkO1E/section/nByvRL).

Whereas the recommendations remain unchanged, the evidence summary available via MAGICapp for corticosteroids was updated before the fifth iteration of the living guideline. The baseline risk estimates for mortality are now based on the WHO SOLIDARITY trial (as for other drugs in this guideline) ${ }^{4}$ rather than the initial ISARIC cohort study that likely overestimates current mortality risks at the global level. ${ }^{29}$ This update was also needed to inform the baseline risk for mortality in the evidence summary informing the strong recommendation for IL-6 inhibitors in addition to standard care for patients with severe or critical covid-19, where

corticosteroids provide a relative reduction in mortality by $21 \%$.

Recommendation 1: We recommend treatment with systemic corticosteroids for patients with severe or critical covid-19 (strong recommendation).

Balance of benefits and harms-Ultimately, the GDG made its recommendation on the basis of a 28 -day mortality reduction of $3.4 \%$ in severe or critical covid-19 combined (moderate certainty). Systemic corticosteroids probably reduce the need for mechanical ventilation (moderate certainty).

Overall, the GDG has reasonable certainty that the adverse effects, when considered together, are sufficiently limited in importance and frequency, and suggested that corticosteroids administered in these doses for 7-10 days are not associated with an increased risk of adverse events, beyond likely increasing the incidence of hyperglycaemia and hyponatremia (both moderate certainty). In contrast with new agents proposed for covid-19, clinicians have vast experience administering systemic corticosteroids, and the GDG was reassured by their overall safety profile.

Values and preferences-The GDG took an individual patient perspective to values and preferences but, given the burden of the pandemic for healthcare systems globally, also placed a high value on resource allocation and equity. The benefits of corticosteroids on mortality were deemed of critical importance to patients, with little or no anticipated variability in their preference to be offered treatment if severely ill from covid-19.

Applicability-Applicability is less clear for populations that were under-represented in the considered trials, such as children, patients with tuberculosis, and those who are immunocompromised. In considering potential contraindications to short term systemic corticosteroids in such patients, clinicians must determine if they warrant depriving a patient of a potentially lifesaving therapy. Clinicians should exercise caution in use of corticosteroids in patients with diabetes or underlying immunocompromise. The GDG was confident that clinicians using these guidelines would be aware of additional potential side effects and contraindications to systemic corticosteroid therapy, which may vary geographically in function of endemic microbiological flora.

Acceptability and practical issues-The ease of administration, the relatively short duration of a course of systemic corticosteroid therapy, and the generally benign safety profile of systemic corticosteroids administered for up to 7-10 days led the GDG to conclude that the acceptability of this intervention was high. Practical issues are summarised in detail on MAGICapp.

Resource implications, feasibility, equity, and human rights-Systemic corticosteroids are low cost, easy to administer, and readily available globally. Dexamethasone and prednisolone are among the most commonly listed medicines in national essential medicines lists; listed by $95 \%$ of countries. Accordingly, systemic corticosteroids are among a relatively small number of interventions for covid-19 that have the potential to reduce inequities and improve equity in health. Those considerations influenced the strength of this recommendation.

Recommendation 2: We suggest not to use systemic corticosteroids for patients with non-severe covid-19 (conditional or weak recommendation).

Balance of benefits and harms-Systemic corticosteroids may increase the risk of 28-day mortality (low certainty).

Values and preferences-The conditional recommendation was driven by likely variation in patient values and preferences. The GDG judged that most individuals with non-severe illness would decline systemic corticosteroids. However, many may want them after shared decision making with their treating physician.

Applicability-Several specific circumstances were considered.

- Systemic corticosteroids should not be stopped for patients with non-severe covid-19 who are already treated with systemic corticosteroids for other reasons (such as patients with chronic obstructive pulmonary disease or chronic autoimmune disease).

- If the clinical condition of patients with non-severe covid-19 worsens (that is, increase in respiratory rate, signs of respiratory distress or hypoxaemia) they should receive systemic corticosteroids (see recommendation 1).

- Pregnancy: antenatal corticosteroid therapy may be administered for pregnant women at risk of preterm birth from 24 to 34 weeks' gestation when there is no clinical evidence of maternal infection 
and adequate childbirth and newborn care are available. In cases where the woman presents with mild or moderate covid-19, the clinical benefits of antenatal corticosteroid might outweigh the risks of potential harm to the mother. In this situation, the balance of benefits and harms for the woman and the preterm newborn should be discussed with the woman to ensure an informed decision, as this assessment may vary depending on the woman's clinical condition, her wishes and those of her family, and available healthcare resources.

- Endemic infections that may worsen with corticosteroids should be considered. For example, for Strongyloides stercoralis hyper-infection associated with corticosteroid therapy, diagnosis or empiric treatment may be considered in endemic areas if steroids are used.

Resource implications, feasibility, equity, and human rights-To help guarantee access to systemic corticosteroids for patients with severe or critical covid-19, it is reasonable to avoid their administration to patients who, given the current evidence, do not seem to derive any benefit from this intervention.

\section{Specific uncertainties, emerging evidence, and future research}

Remaining uncertainties include effects on:

- Patients with non-severe covid-19 (that is, pneumonia without hypoxaemia).

- Immunity and the risk of a subsequent infection, which may affect the risk of death after 28 days.

- By different steroid preparation, dosing, and optimal timing of drug initiation.

How this living guideline was created (see MAGICapp for full details https://app.magicapp.org/\#/guideline/nBk01E)

Standards, methods, and processes for living and trustworthy guidance The Guideline Development Group (GDG) produced the recommendations following standards for trustworthy guideline development using the GRADE (Grading of Recommendations Assessment, Development and Evaluation) approach, in compliance with the WHO Handbook for

Guideline Development 2nd Edition, 30 the Institute of Medicine, and the

Guideline International Network (G-I-N). ${ }^{31}$

\section{Selection and support of the GDG}

WHO convened a Guideline Development Group (GDG) with content experts (clinicians, methodologists, scientists) and patients who previously had covid-19. The methods chair (methodological expertise) and a clinical chair (content expertise) guided the GDG discussions. GDG members were invited by WHO, with the aim of achieving gender, geography, expertise, and patient representation balance as well as relevant technical and clinical expertise. The WHO technical unit collected and managed declarations of interests (DOIs) and found no GDG member, chair, or systematic review team member to have a conflict of interest. The GDG aimed to create a recommendation based on consensus with a provision for voting that proved unnecessary for this recommendation. Co-chairs were not eligible to vote in this setting. For recommendations revised or added in the current iteration, there was no need for voting. Guideline perspective, outcomes, and values and preferences The target audience for this guidance consists of clinicians, patients, and healthcare decision makers. The GDG defined covid-19 by clinical severity (box 2). The GDG considered an individual patient perspective, but also took account of contextual factors (such as resources, feasibility, acceptability, and equity) to accommodate global re-use and adaptation for countries and healthcare systems, and to recognise system challenges in implementing recommendations.

There were insufficient published data to provide the GDG with an evidence-based description of patient experiences, or values and preferences regarding treatment decisions for covid-19 drug treatments.
The GDG therefore relied on their own judgments of what well informed patients would value after carefully balancing the benefits, harms, and burdens of treatment. These judgments on values and preferences were also informed through the experiences of former patients with covid-19, represented in the GDG.

The GDG agreed that the following values and preferences would be representative of those of typical well informed patients:

- Most patients would be reluctant to use a treatment for which the evidence left high uncertainty regarding effects on the outcomes they consider important. This was particularly so when evidence suggested treatment effects, if they exist, are small and the possibility of important harm remains.

- In an alternative situation with larger benefits and less uncertainty regarding both benefits and harms, more patients would be inclined to choose the treatment.

\section{Sources of evidence}

To create recommendations, the GDG relied on evidence synthesised in two living network meta-analyses coordinated by MAGIC. ${ }^{6} 7$

\section{Derivation of absolute effects for drug treatments}

For patients with non-severe illness, we used the median of the control arm of the RCTs that contributed to the evidence. For patients with severe or critical illness, the GDG identified the control arm of the WHO SOLIDARITY trial, performed across a wide variety of countries and geographical regions, as representing the most relevant source of evidence for baseline risk estimates for mortality and mechanical ventilation. ${ }^{4}$ Systemic corticosteroids now represent standard of care in patients with severe or critical covid-19 (see strong recommendation issued by WHO in September 2020). Therefore, the baseline risk estimates in the evidence summaries for JAK inhibitors, convalescent plasma and IL-6 receptor blockers were adjusted for treatment effects of

corticosteroids for the outcome of mortality and mechanical ventilation. 4 For other outcomes, we used the median of the control arm of the RCTs that contributed to the evidence. Baseline risks, and thus absolute effects, may vary significantly geographically and over time. Thus, users of this guideline may prefer estimating absolute effects by using local event rates. Recommended combinations of treatments are based on direct comparisons from trials demonstrating additional benefit, such as adding baricitinib or interleukin- 6 receptor blockers to systemic corticosteroids in patients with severe or critical covid-19. In patients with non-severe covid-19 the absence of direct comparisons from RCTs necessitate indirect comparisons from the living network meta-analysis to inform judgments made about alternative treatment options.

\section{How patients were involved in the creation of this article}

The GDG included patients who previously had covid-19. Their perspectives were crucial in considering the values and preferences associated with the various treatments.

\section{AUTHOR AFFILIATIONS}

Université de Sherbrooke, Centre de recherche due CHU de Sherbrooke, Quebec, Canada

Department of Health Research Methods, Evidence and Impact, McMaster University, Hamilton, Ontario, Canada

Department of Medicine, McMaster University, Hamilton, Ontario, Canada

4 MAGIC Evidence Ecosystem Foundation, Oslo, Norway

Division of General Internal Medicine \& Division of Clinical Epidemiology, University Hospitals of Geneva, Geneva, Switzerland

6 World Health Organization, Geneva, Switzerland

National Center for Infectious Diseases, Singapore 
8

, London, UK

Ministry of Health and Population, Cairo, Egypt

Fluminense Federal University, Brazil

11

Mediciti Hospital, Nepal

12

Antioquia University Medellin, Colombia

13

Geneva University Hospital, Switzerland

Infection Division, Department of Pulmonology and Respiratory Medicine, Faculty of Medicine Universitas Indonesia

15

University of California, San Francisco, USA

16

Department of Anesthesia and Intensive Care Medicine, Humanitas Clinical and Research Center

Christian Medical College, Vellore, India

Adult Infectious Disease Centre, University Teaching Hospital, Lusaka, Zambia

Department of Infectious Diseases, Hanoi Medical University, Hanoi, Vietnam

20

University of Gent, Belgium

Peking Union Medical College Hospital, Beijing, China

Department of Pediatrics, Cumming School of Medicine, University of Calgary, Canada

23

Division of Emergency Medicine, Faculty of Medicine and Health Sciences, Stellenbosch University, Cape Town, South Africa

24

United States

University of Alberta, Canada

Royal Melbourne Hospital and Epworth Healthcare, Melbourne, Australia

Ziauddin University, Karachi, Pakistan

28

St Thomas' Hospital, London, UK

Aga Khan University, Pakistan

All India Institute of Medical Sciences, New Delhi, India

McMaster University, Canada (alumnus)

32

Sungkyunkwan University School of Medicine, Samsung Medical Center, Seoul, Republic of Korea

Department of Paediatrics and Emergency Medicine, University of British Columbia, Vancouver, Canada

34

St George's University of London, UK

35

Department of Anaesthesia and Critical Care, College of Health Sciences, Makerere University, Kampala, Uganda

36

HCOR Hospital do Coracao, Sao Paulo, Brazil

Department of Paediatrics, All India Institute of Medical Sciences, India

Zimbabwe

39

Emergency Medical Services, Faculty of Medicine, Tunis, Tunisia

Groote Schuur Hospital, University of Cape Town, South Africa
41

Clinical Scientific Institutes Maugeri, Italy

Alcivar Hospital in Guayaquil, Ecuador

43

Sheikh Shakhbout Medical City, Abu Dhabi Central Research Institute of Epidemiology of Rospotrebnadzor, Moscow, Russia

45

Division of Pulmonary and Critical Care Medicine, David Geffen School of Medicine, University of California Los Angeles, Los Angeles, California, USA

Aga Khan University, Karachi, Pakistan

47

National Institute of Tuberculosis and Respiratory Diseases, New Delhi, India

48

Guy's and St Thomas' NHS Foundation Trust, London, UK

49

St. George’s University Hospital, UK

50

Shanghai Public Health Clinical Center, Fudan University, Shanghai, China

51

University of Colombo, Sri Lanka

University of Sao Paulo, Brazil

Charité - Universitätsmedizin Berlin, Germany

54

University of North Carolina, USA

Faculty of Medicine Andres Bello University, Indisa Clinic, Santiago, Chile

56

Influenza Division, U.S. Centers for Disease Control and Prevention, USA

King's College, London, UK

58

University of Stellenbosch, South Africa

59

Ministry of Health, Sri Lanka

60

Infectious Diseases Data Observatory (IDDO), Centre for Tropical Medicine and Globa Health, Nuffield Department of Medicine, University of Oxford, Oxford, UK

61

Evidence Based Social Science Research Centre, School of Public Health, Lanzhou University, Lanzhou, China and the Department of Social Medicine and Health Management, School of Public Health, Lanzhou University, Lanzhou, China

62

Department of Molecular and Clinical Pharmacology, University of Liverpool, Liverpool, UK

HCOR Research Institute, Hospital do Coracao, Sao Paulo, Brazil; Pulmonary Division, Heart Institute (InCor), University of Sao Paulo, Sao Paulo, Brazil

Royal Free London NHS Foundation Trust, UK Department of Medicine, Lovisenberg Diaconal Hospital Trust, Oslo, Norway

Not Guideline Development Group member; resource for methodology, systematic review, and content support

co-senior author

Funding: Bill \& Melinda Gates Foundation, Norwegian Directorate of Public Health and Germany provided funding for this guideline, with MAGIC providing pro-bono contributions and support to WHO in the context of the covid-19 pandemic

Competing interests: All GDG members have completed the WHO interest disclosure form. All authors have completed the BMJ Rapid Recommendations interest of disclosure form. The WHO, MAGIC and The BMJ judged that no GDG member or co-chair had any financial conflict of interest. Professional and academic interests are minimised as much as possible, while maintaining necessary expertise on the GDG to make fully informed decisions. MAGIC and TheBM/ assessed declared interests from other co-authors of this publication and found no relevant conflicts of interests. 
Provenance and peer review: This publication was commissioned by The BM/ in partnership with WHO and the MAGIC Evidence Ecosystem Foundation, in the context of the BMJ Rapid Recommendations. Pre-publication internal and external peer-review managed by WHO, and internal review at The BMJ. Post-publication review through rapid responses on bmj.com and through MAGICapp.

We thank all the following collaborators who contributed to this endeavour, as detailed in the WHO guidance (see link in box 1)

- World Health Organisation (WHO) Secretariat for Therapeutics and COVID-19

- External reviewers for WHO

We also thank

- The living systematic review and network meta-analysis team, led by investigators Reed Siemieniuk and Romina Brignardello-Petersen at McMaster University, Canada

- BMJ editorial team of Greg Cotton (technical editor), Cat Chatfield (head of education), and Will Stahl-Timmins (data graphics designer) for their work on this living guideline publication in The BMJ

- Lien Tran, Lorenzo Arena, Kasia Stepniewska, and Philippe Guerin for providing up to date data on registered and ongoing trials from the Infectious Diseases Data Observatory (IDDO) living systematic review of covid-19 clinical trial registrations (https://www.iddo.org/covid-19/live-systematic-reviewtrials). Data extraction is supported by Fiona Caldwell, Vitalis Feteh, Gerald Makuka, Roland Ngu, and Chinwe Ogbonnaa-Njoku.

- Andrew Owen (University of Liverpool, UK) for contributions on pharmacological considerations and analyses

1 Maguire BJ, Guérin PJ. A living systematic review protocol for COVID-19 clinical trial registrations. Wellcome Open Res 2020;5:. doi: 10.12688/wellcomeopenres.15821.1 pmid: 32292826

2 Angus DC, Derde L, Al-Beidh F, etalWriting Committee for the REMAP-CAP Investigators. Effect of hydrocortisone on mortality and organ support in patients with severe COVID-19: the REMAP-CAP COVID-19 corticosteroid domain randomized clinical trial. JAMA 2020;324:-29. doi: 10.1001/jama.2020.17022 pmid: 32876697

3 Horby P, Lim WS, Emberson JR, etalRECOVERY Collaborative Group. Dexamethasone in hospitalized patients with Covid-19. N Engl J Med 2021;384:-704. doi: 10.1056/NEJMoa2021436 pmid: 32678530

4 Pan H, Peto R, Henao-Restrepo AM, etalWHO Solidarity Trial Consortium. Repurposed antiviral drugs for Covid-19 - Interim WHO Solidarity Trial results. N Engl / Med 2021;384:-511. doi: 10.1056/NEJMoa2023184 pmid: 33264556

5 Lundgren JD, Grund B, Barkauskas CE, etalACTIV-3/TICO LY-CoV555 Study Group. A neutralizing monoclonal antibody for hospitalized patients with Covid-19. N Engl / Med 2021;384:-14. doi: 10.1056/NEJMoa2033130 pmid: 33356051

6 Siemieniuk RA, Bartoszko JJ, Ge L, etal. Drug treatments for covid-19: living systematic review and network meta-analysis. BM/2020;370:. doi: 10.1136/bmj.m2980 pmid: 32732190

7 Siemieniuk RAC, Bartoszko JJ, Díaz Martinez JP, etal. Antibody and cellular therapies for treatment of covid-19: a living systematic review and network meta-analysis. BMJ2021;374:. doi: 10.1136/bmj.n2231 pmid: 34556486

$8 \quad$ World Health Organization. Clinical management of COVID-19: interim guidance. 2020. https://www.who.int/publications/i/item/clinical-management-of-covid-19.

9 Wynants L, Van Calster B, Collins GS, etal. Prediction models for diagnosis and prognosis of covid-19: systematic review and critical appraisal. BMJ2020;369: doi: 10.1136/bmi.m1328 pmid: 32265220

10 United States Food and Drug Administration. Fact sheet for healthcare providers: emergency use authorization for PAXLOVID. 2022. https://www.fda.gov/media/155050/download.

11 Owen DR, Allerton CMN, Anderson AS, etal. An oral SARS-CoV-2 $\mathrm{M}^{\text {pro }}$ inhibitor clinical candidate for the treatment of COVID-19. Science 2021;374:-93.

doi: 10.1126/science.abl4784 pmid: 34726479

12 Abdelnabi R, Foo CS, Jochmans D, etal. The oral protease inhibitor (PF-07321332) protects Syrian hamsters against infection with SARS-CoV-2 variants of concern. Nat Commun 2022;13:. doi: 10.1038/s41467-022-28354-0 pmid: 35169114

13 Takashita E, Yamayoshi S, Simon V, etal. Efficacy of antibodies and antiviral drugs against omicron BA.2.12.1, BA.4, and BA.5 subvariants. N Engl J Med 2022;387:-70. doi: 10.1056/NEJMc2207519 pmid: 35857646

14 Vangeel L, Chiu W, De Jonghe S, etal. Remdesivir, molnupiravir and nirmatrelvir remain active against SARS-CoV-2 omicron and other variants of concern. Antiviral Res 2022;198:105252. doi: 10.1016/j.antiviral.2022.105252 pmid: 35085683

15 University of Liverpool. COVID-19 drug interactions. https://www.covid19-druginteractions.org/checker.

16 RECOVERY Collaborative Group. Baricitinib in patients admitted to hospital with COVID-19 (RECOVERY): a randomised, controlled, open-label, platform trial and updated meta-analysis. Lancet 2022;400:-68. doi: 10.1016/S0140-6736(22)01109-6 pmid: 35908569

17 Owen A, Diaz JV, Guyatt G, etal. WHO Living Guidelines on antivirals for COVID-19 are evidence-based. Lancet2023;400:-8. . doi: 10.1016/S0140-6736(22)02306-6 pmid: 36372074
18 Sheahan TP, Sims AC, Zhou S, etal. An orally bioavailable broad-spectrum antiviral inhibits SARS-CoV-2 in human airway epithelial cell cultures and multiple coronaviruses in mice. Sci Transl Med 2020;12:eabb5883. doi: 10.1126/scitransImed.abb5883 pmid: 32253226

19 Tao S, Zandi K, Bassit L, etal. Comparison of anti-SARS-CoV-2 activity and intracellular metabolism of remdesivir and its parent nucleoside. Curr Res Pharmacol Drug Discov 2021;2:100045. doi: 10.1016/j.crphar.2021.100045 pmid: 34870151

20 Rosenke K, Hansen F, Schwarz B, etal. Orally delivered MK-4482 inhibits SARS-CoV-2 replication in the Syrian hamster model.Research Square 2020doi: 10.21203/rs.3.rs-86289/v1

21 Cox RM, Wolf JD, Plemper RK. Therapeutically administered ribonucleoside analogue MK-4482/EIDD-2801 blocks SARS-CoV-2 transmission in ferrets. Nat Microbiol 2021;6:-8. doi: 10.1038/s41564-020-00835-2 pmid: 33273742

22 Abdelnabi R, Foo CS, Kaptein SJF, etal. The combined treatment of molnupiravir and favipiravir results in a potentiation of antiviral efficacy in a SARS-CoV-2 hamster infection model. EBioMedicine 2021;72:103595. doi: 10.1016/j.ebiom.2021.103595 pmid: 34571361

23 Abdelnabi R, Foo CS, De Jonghe S, Maes P. Weynand B, Neyts J. Molnupiravir inhibits replication of the emerging SARS-CoV-2 variants of concern in a hamster infection model. J Infect Dis 2021;224:-53. doi: 10.1093/infdis/jiab361 pmid: 34244768

24 Prince T, Donovan-Banfield I, Goldswain $\mathrm{H}$, etal. Antiviral activity of molnupiravir precursor NHC against variants of concern (VOCS) and its therapeutic window in a human lung cell model.bioRxiv 2021doi: 10.1101/2021.11.23.469695

25 Vangeel L, De Jonghe S, Maes P, etal. Remdesivir, molnupiravir and nirmatrelvir remain active against SARS-CoV-2 omicron and other variants of concern.bioRxiv 2021doi: 10.1101/2021.12.27.474275

26 Shankar-Hari M, Vale CL, Godolphin PJ, etalWHO Rapid Evidence Appraisal for COVID-19 Therapies (REACT) Working Group. Association between administration of IL-6 antagonists and mortality among patients hospitalized for COVID-19: a meta-analysis. JAMA 2021;326:-518. doi: 10.1001/jama.2021.11330 pmid: 34228774

27 Elgazzar A, Hany B, Youssef SA, etal. Efficacy and safety of ivermectin for treatment and prophylaxis of COVID-19 pandemic. ResearchSquare 2021; .

28 Reardon S. Flawed ivermectin preprint highlights challenges of COVID drug studies. Nature 2021;596:-4. doi: 10.1038/d41586-021-02081-w pmid: 34341573

29 Docherty AB, Harrison EM, Green CA, etallSARIC4C investigators. Features of 20133 UK patients in hospital with covid-19 using the ISARIC WHO Clinical Characterisation Protocol: prospective observational cohort study. BMJ 2020;369:. doi: 10.1136/bmi.m1985 pmid: 32444460

30 World Health Organization. Handbook for guideline development. 2008. https://www.who.int/publications/guidelines/handbook_2nd_ed.pdf?ua=1.

31 Qaseem A, Forland F, Macbeth F, Ollenschläger G, Phillips S, van der Wees PBoard of Trustees of the Guidelines International Network. Guidelines International Network: toward international standards for clinical practice guidelines. Ann Intern Med 2012;156:-31. doi: 10.7326/0003-4819-156-7-201204030-00009 pmid: 22473437

Main infographic: Summary of recommendations and evidence 